\title{
3D printed elastomeric polyurethane: Viscoelastic experimental characterisations and constitutive modelling with nonlinear viscosity functions
}

\author{
Mokarram Hossain $^{\mathrm{a}, *}$, Rukshan Navaratne ${ }^{\mathrm{b}}$, Djordje Perića \\ ${ }^{a}$ Zienkiewicz Centre for Computational Engineering, College of Engineering, Swansea University, SA1 8EN, United Kingdom \\ ${ }^{b}$ Aerospace Engineering Department, University of South Wales, Pontypridd, Cardiff, United Kingdom
}

\begin{abstract}
Digital Light Synthesis (DLS) technology creates ample opportunities for making 3D printed soft polymers for a wide range of grades and properties. In DLS, a 3D printer uses a continuous building technique in which the curing process is activated by an ultra-violet (UV) light. In this contribution, EUP40, a recently invented commercially available elastomeric polyurethane (EPU) printed by the DLS technology, is experimentally characterised. For characterizing the mechanical properties, an extensive viscoelastic experimental study on the digitally printed EPU taking the strain rate-dependence are conducted. The study reveals a significant time-dependency on its mechanical responses. Moreover, the material demonstrates noticeable nonlinear viscosities that depend on strain and strain rates. Based on the experimental findings for the printed elastomer, a large strain viscoelastic model is devised where evolution laws are enhanced by strain and strain rate-dependent nonlinear viscosities. Following identifications of relevant material parameters, we validate the model with the experimental data that show its good predictability. Such an extensive experimental study along with a constitutive model will help in designing and simulating more complex cellular and structured metamaterials using 3D printed elastomeric polyurethanes.
\end{abstract}

Keywords: Digital Light Synthesis (DLS), Elastomeric Polyurethane (EPU), 3D printing, Additive manufacturing, Viscoelastic characterization

\section{Introduction}

The additive manufacturing (AM) or 3D printing is a new paradigm revolutionising the concepts of producing multi-characteristics, multi-purpose, complex metamaterials. Now ideas and designs can easily be converted in developing prototypes directly thanks to the 3D printing. Therefore, AM has been drawing 5 unprecedented and ever-increasing interests from industry and academia alike [1,9,33]. A wide range of techniques have continuously been evolved resulting in high print quality, complex, and intricate geometries which are simply not possible with classical manufacturing techniques. AM, combined with complex and rational designs, helps us in creating ultra-stiff and ultra-light parts within a material, see $[1,36,46]$. According to a recent report [63], almost half of the additively manufactured digital materials are soft polymers. 10 Polymeric materials are nowadays being produced by various 3D printers with a wide range of properties. In the 3D printing of polymeric materials, an UV light is used for the curing process which can be produced multi-materials in complex shapes. Among other 3D printing companies, Stratasys, Carbon3D, ACEO3D,

\footnotetext{
*mokarram.hossain@swansea.ac.uk

Email addresses: mokarram. hossaineswansea. ac.uk (Mokarram Hossain), rukshan.navaratneesouthwales.ac.uk (Rukshan Navaratne), d.periceswansea.ac.uk (Djordje Perić)
} 
RepRap, Protolabs, Materialise, etc produce varieties of soft polymeric materials which can sustain extremely large deformations. The soft photo-curing resins could be acrylics, silicones, and polyurethanes, while several AM techniques such as Inkjet printing, Drop-on Demand (DOD), Stereolithography Apparatus (SLA), Digital Light Processing (DLP), and Continuous Liquid Interface Production (CLIP)/Digital Light Synthesis (DLS) have been widely used. Among other processes for soft polymers and their composites, the DLS (also known as CLIP) helps to reduce the design and fabrication processes from several hours to a few minutes or even less $[33,55,56]$.

Experimental characterization and computational modelling of additively manufactured hard materials such as metals and their alloys are active fields of current research [9]. Simonelli et al. [53] proposed a novel approach to jet molten metals at high-temperatures $\left(>1000^{\circ} \mathrm{C}\right)$ to enable the direct digital additive fabrication of micro-to macro-scale objects. Lizarazu et al. [34] conducted an experimental study on wire arc addiby the AM process that involves several heating and cooling cycles. Very recently, Ghorbanpour et al. [17] experimentally characterized the strength and microstructural evolution of superalloy Inconel 718 (IN718) as a function of the initial microstructure created via direct metal laser melting additive manufacturing technology. Therein, they measured stress-strain curves in tension and compression from room temperature to $550^{\circ} \mathrm{C}$ and crystallographic texture is characterized using neutron diffraction. In addition, they developed crystal plasticity modeling of anisotropy, tension-compression asymmetry, and texture evolution of the additively manufactured metals. However, despite many applications of digitally printed soft materials such as polymers and their composites, comprehensive mechanical experimental characterisations at large strain of some widely-known polymeric materials are limited in the literature. Only small strain-based characterizations, such as determination of various moduli including shear modulus, bulk modulus, loss modulus etc, have been performed, see [5]. For instance, Lumpe et al. [32] measured the tensile strength of VeroWhite (Stratasys Ltd.). They mixed two materials of distinct hardnesses, one rigid-brittle and another rubbery. Very recently, Mueller et al. [39] performed experiments to understand the mechanical properties of Inkjet-based 3D printed structures. Reichl and Inman [45] characterized viscoelastic properties of several 3D printed soft polymers by determining the complex moduli that depend on the frequency and temperature. Note that these moduli provide inadequate and inaccurate information for viscoelastic materials in finite strain regime. Very recently, Hossain and Liao [20] conducted an extensive thermo-viscoelastic experimental study on a DLS-printed soft silicone polymer. Moreover, based on the experimental data, they developed a constitutive framework to capture the thermo-viscoelastic behaviour at large strains.

3D printed soft polymers are promising materials in soft robotics [8, 10, 38, 52, 62]. In order to develop reliable and accurate mathematical models for such materials, experimental characterization of their viscoelastic behaviour, especially relaxation and strain rate-dependent phenomena, are very much essential, see $[11,18,28,31]$. To fulfil the gaps in experimental characterizations of digitally manufactured acrylic (Tangoflex series) polymers, very recently Slesarenko and Rudykh [54], Liu and Li [31], Morris et al. [40] performed strain rate-dependent experiments at large strains. However, these pioneering studies lack by not considering some classical experiments, such as loading-unloading cyclic tests, stress softening tests to identify Mullins effects, single-and multi-step relaxation tests etc. Aforementioned major experiments are crucial in developing a comprehensive understanding of the 3D printed viscoelastic soft polymeric materials at finite strains. Note that experimental characterization of FDM (fused deposition modelling)-based 3D printed thermoplastic polymers and their composites have been actively investigated in the literature $[1,14,46]$. However, digitally-printed relatively rigid materials, such as thermoplastics have reduced appli- 
cations in flexible structures including soft robotics and programable metamaterials.

As a result of high elasticity combined with high impact and abrasion resistances, polyurethanes and their variants such as polyurea have significant applications such as ski boots, gaskets, seals, interlayers in windshield glasses [12, 16, 27, 58]. Transparent elastomeric polyurethane is a promising candidate in the design concept of transparent armour. Moreover, the elastomeric polyurethane is a major candidate in recently emerged magneto-rheological elastomer, a fast growing composite polymeric material used in soft robotics and vibration control, see Bastola and Hossain [4] for an exhaustive review. Hence, the understanding of the mechanical behaviour of polyurethanes gains considerable attention in recent years. A significant research effort has been invested on experimental characterizations to assess their mechanical properties as well as to model constitutive behaviour. Some earliest works in the area of mechanical characterization of polyurethanes and polyurea are due to Qi et al. [44] and Yi et al. [58], wherein they performed tests from Fan et al. [16] performed extensive experiments on elastomeric polyurethanes to identify the transition region between the rubbery-like and the glassy-like behaviour using low to very high strain rates. Johlitz et al. [23] performed viscoelastic experiments, particularly rate-dependent tests at small strain-rates, for an elastomeric polyurethane and developed a constitutive model. Note that thermoplastic polyurethanes and polyurea are chemically different from elastomeric polyurethanes (EPU). Moreover, aforementioned EPUs and TPUs are either room temperature-cured or thermally-cured polymers at an elevated temperature whose properties are significantly different from 3D printed EPUs which are manufactured using a photo-curing process at room temperature.

3D printed EPU has been first made commercially available by Carbon3D, a 3D printing company mainly devoted producing soft polymers. EPU, digitally printed using the Digital Light Synthesis (DLS) technique, is a comparatively high elastic, high tear resistant, and more resilient elastomer [6]. EPU40 ${ }^{\mathrm{TM}}$ (Carbon3D, USA) is a member of the elastomeric material family that has an excellent combinations of tear strength, energy return, and a high elongation at break that makes it perfect for cushioning, impact absorption, vibration isolation, gaskets, and seals. It is especially well-suited for producing elastomeric lattices to create foam-like products. Moreover, EPU-based polymers have gained popularity as compliant implant materials in the arena of biomedical engineering [57]. The AM provides new and ever-expanding applications in bio-sciences where it helps to create more complex architectures for tissue scaffolding and patient specific implants using light-cured based techniques [35]. Very recently, Miller et al. [35] quantify the effects of various printing factors on the mechanical behaviour of elastomeric EPUs (EPU40 and EPU41 of Carbon3D). However, an extensive viscoelastic characterization and material modelling has not been reported yet for the 3D printed EPU.

The contribution of this study has two specific aims. At first, a comprehensive viscoelastic experimental study of a recently invented DLS-based EPU (EPU40) is demonstrated taking into account some classical experiments suitable for understanding a polymeric material at finite strains. These experiments are stresssoftening tests, quasi-static tests, cyclic tests, single- and multi-step relaxation tests. Experimental results show a significant nonlinear viscosity that depends on strains and strain rates. Secondly, we develop a thermodynamically consistent geometrically nonlinear viscoelastic numerical model for the digitally printed polymer taking into account the experimentally-observed nonlinear viscosity. Since, experimental results are obtained using a uniaxial mode of deformation, we reformulate the three-dimensional viscoelastic constitutive model to one-dimensional forms. Afterwards, all parameters of the model are identified using an 


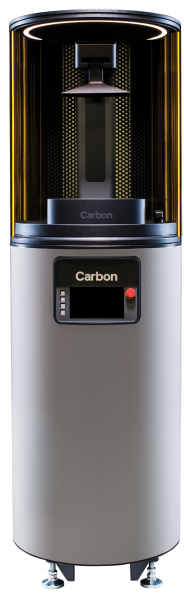

(a)

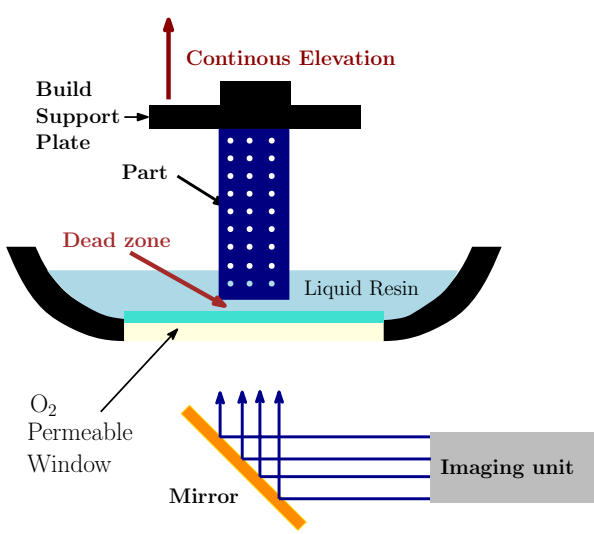

(b)

Figure 1. Carbon3D DLS technology : (a) Carbon3D's latest 3D printer CarbonM2, (b) a sketch describing the basic functionality of the DLS technology

optimisation procedure. In the final section, the constitutive equations are verified with a new set of experimental data.

The manuscript has six Sections. In Sec. 2, a short account of sample preparations describing the experimental procedures in our laboratory is presented. In Sec. 3, in addition to the descriptions of each type of characterization procedure, all findings are presented with necessary analyses. In an effort to devise experimentally-driven constitutive frameworks for the 3D printed EPU, a thermodynamically consistent obtained from uniaxial homogeneous types of experiments, relevant equations appearing in the model are reduced to one-dimensional forms which are required for finding appropriate parameter sets. Sec. 5 deals with the model validation. Finally, concluding remarks close the paper in Sec. 6 with an outlook for future works.

\section{Experimental details}

\subsection{A short note on Digital Light Synthesis (DLS) process}

Digital Light Synthesis or DLS (previously known as Continuous Liquid Interface Production or CLIP) invented by DeSimone and co-workers [56] radically changes traditional time-consuming layer-by-layer or dot-by-dot building processes, such as Stereolithography Apparatus (SLA) or Digital Light Projection (DLP) for making soft polymers and their composites. 3D printing of an object under the DLS process starts at the bottom. Hence, it is called a bottom-up building approach. The printer has several parts, namely an oxygen inhibited dead-zone and an oxygen-permeable curing window, see Fig. 1(b). The curing window is an extremely thin layer, one third of a human hair. Moreover, it has a low refractive index and outstanding optical clarity. The dead-zone prevents any attachment of the 3D part to the curing window. When oxygen 


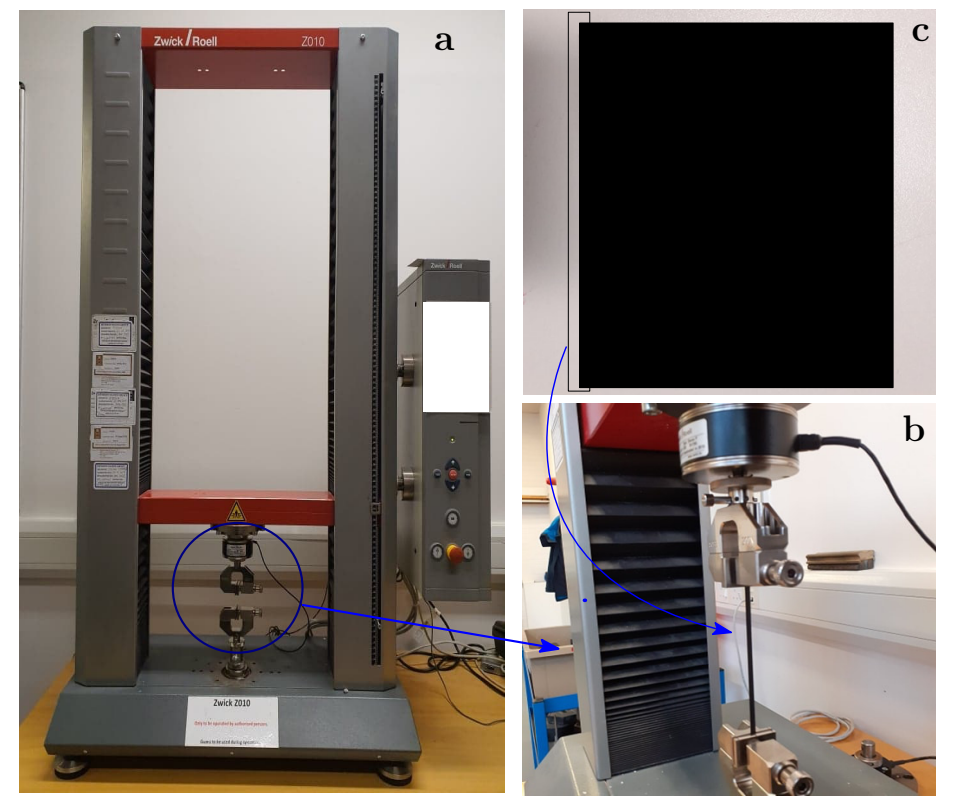

Figure 2. A complete experimental set-up: (a) The main framework of the Zwick/Roell Z010 machine highlighting the gripping section in a circle, (b) A closeup look of the gripping system along with an elongated specimen, (c) A large 3D printed rectangular EPU40 sheet (black color) of $100 \mathrm{~mm} \times 120 \mathrm{~mm}$ x $1 \mathrm{~mm}$ (width $\mathrm{x}$ length $\mathrm{x}$ thickness)

permeates through the window it creates the dead-zone, of uncured resin between the window and the printing part. The dead-zone is the area mainly responsible for Carbon3D's remarkable performance with isotropic 3D printed objects. In the zone, oxygen prohibits lightening curing the resins situated closest to the window, therefore allowing the continuous flow of liquid resins beneath the part [55]. A Carbon (M2) 3D printer has high-resolution pixels of 75 micrometer [6].

\subsection{Sample preparation}

For the viscoelastic experimental characterizations of the 3D printed elastomeric polyurethane (EPU) outlined in this paper, among other brands, EPU40 produced by Carbon3D ${ }^{\mathrm{TM} 1}$ CarbonM2 printer is selected. CarbonM2 (Fig. 1(a)) uses the DLS technology for manufacturing various soft polymers. For the current study, we purchased necessary samples from Paragon Rapid Technologies (UK), a local Production Network of the Carbon3D. In preparing specimens for uniaxial homogeneous tests, two techniques have widely been used in the area of soft polymers experimentation. First option is the so-called dog-bone samples that significantly reduce the grippers' (edge) effects due to the presence of enhanced areas at sample ends $[14,59]$. Second alternative route is the rectangular sample, in which a length to width ratio $\approx 10: 1$ is maintained throughout the sample length $[18,28,29,30,61]$. In order to reduce the cost for the sample production, the second option for the specimen preparation is adopted in the current study. For that, a CAD (Computer Aided Design) geometry of $1 \mathrm{~mm}$ uniform thickness with a rectangular area of $100 \mathrm{~mm} \times 120$ mm (Fig. 2c, black color) is created and exported to a STL (Standard Tessellation Language) file format. Afterwards, slices of $5 \mathrm{~mm}$ wide and $120 \mathrm{~mm}$ long are made from the big sample, see Fig. 2c. Again a slice is divided into two pieces, $5 \mathrm{~mm}$ wide and $60 \mathrm{~mm}$ long each. Finally, a rectangular sample is created with

\footnotetext{
${ }^{1}$ https: //www. carbon3d.com
} 
$5 \mathrm{~mm} \times 60 \mathrm{~mm} \times 1 \mathrm{~mm}$ (width $\mathrm{x}$ length $\mathrm{x}$ thickness). Within the gripping system, when a sample is placed in the grippers, the gauge is around $40 \mathrm{~mm}$ long and $5 \mathrm{~mm}$ wide. Such a gauge size maintains a 8:1 ratio (length: width) which has widely been considered for a uniaxial homogeneous mode of deformation, see Liao et al. [28, 30]. In order to prepare rectangular samples of $5 \mathrm{~mm}$ x $60 \mathrm{~mm}$ x $1 \mathrm{~mm}$, at first, desired lines are inscribed on the top of the big sample $(100 \mathrm{~mm} \times 120 \mathrm{~mm})$ using a soft ballpoint pen. Afterwards, a sharp trimmer (e.g., Dahle brand) is used to cut a desired sample. A similar sample dimension was taken previously in the case of soft polymers like EPU for testing a homogeneous tensile mode of deformation, see Liao et al. [28, 29, 30], Wissler and Mazza [61]. In order to avoid any time-induced mechanical ageing of the 3D printed EPU, all mechanical experiments are completed within a month of the material printing.

\subsection{Machine preparation}

For a viscoelastic study of the 3D printed EPU, we use a Zwick/Roell (model Z010) machine as shown Fig. 2, in which (a) is the main framework of the machine highlighting the gripping section in a circle, (b) is a closeup view of the gripping system along with an elongated specimen, and (c) is a large 3D printed rectangular EPU40 sheet of $100 \mathrm{~mm} \times 120 \mathrm{~mm}$ x 1 $\mathrm{mm}$ (width x length $\mathrm{x}$ thickness) from which a final sample is cut. A sensor is attached to the machine that can calculate a maximum $\pm 200 \mathrm{~N}$ force within a resolution of $0.001 \mathrm{~N}$. At room temperature, the displacement limit of the machine is $1200 \mathrm{~mm}$. The loading speed of the crosshead can reach $1000 \mathrm{~mm} / \mathrm{min}(\approx 18 \mathrm{~mm} / \mathrm{s})$. The machine interacts with the control computer via the TESTXPERT ${ }^{\circledR}$ user interface software, a built-in Zwick/Roell software. For result presentations, we use the so-called nominal (first Piola-Kirchhoff) stress and the corresponding nominal strain in which the nominal stress (also known as the engineering stress) is calculated by dividing the applied force with the initial (undeformed) cross-sectional area of the sample. Strain rates are the time derivative of the nominal strain (i.e., applied displacement divided by the initial length of the gauge section). The so-called Cauchy stress/ true stress (i.e., the applied force divided by the actual/current cross-sectional area of a sample) is also widely taken in the literature. However, in this study, the nominal stress is chosen because it is relatively easy to measure in our experimental set-up. A good number of data in each interval is extracted using a TESTXPERT ${ }^{\circledR}$ software supplied by Zwick/Roell. Reproducibility is an important issue in any experimental study. Hence, we repeat each test at least five times and average one is taken as the result.

\section{Experimental results}

In this section, experimental results of the 3D printed elastomeric polyurethane (EPU40) polymer are presented. For getting an overview of the mechanical properties of a viscoelastic soft polymer, several classical experiments are essential. These include quasi-static tests, stress-softening tests, strain rate-dependent cyclic tests, single- and multi-step relaxation tests, see Amin et al. [2], Hossain et al. [19]. Note that all experiments in this study are conducted at room temperature $\left(20^{\circ} \mathrm{C}\right)$.

\subsection{Mullins stress-softening behaviour of EPU4O}

Polymeric materials typically demonstrate stress-softening behaviour or the Mullins effect [15]. This means that if a cyclic test is performed on a pre-treated sample, then the stress-strain responses will be different from the one which is not tested or treated previously, i.e., from a virgin sample. Therefore, it is essential to conduct tests to assess whether EPU40 elastomer shows any significant stress-softening behaviour. In order to identify the Mullins effect, two types of loading-unloading cyclic tests are conducted in this study, 


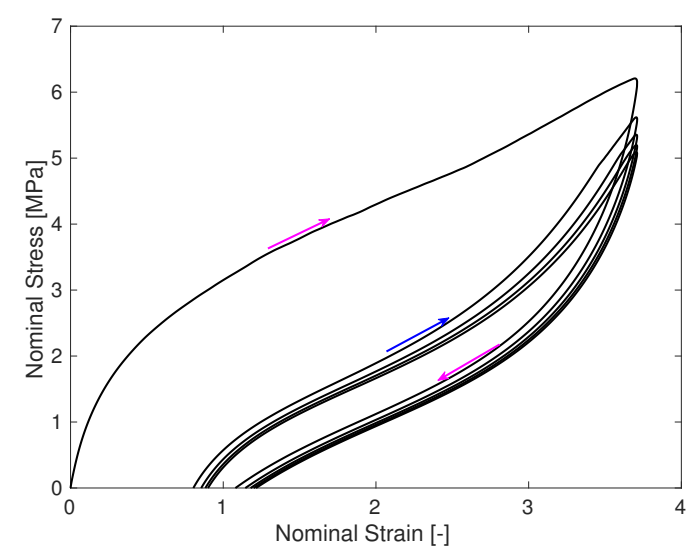

(a)

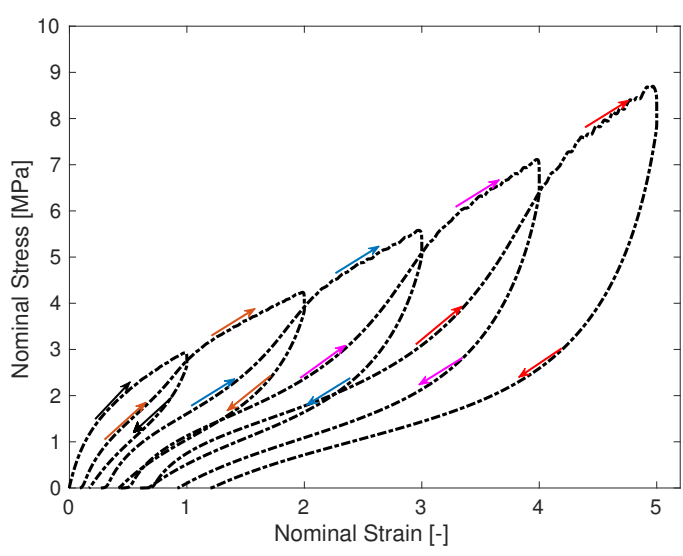

(b)

Figure 3. Mullins stress-softening tests : (a) Repeated cycles at a fixed strain (375\%) and strain rate (0.384 /s) on a virgin sample, (b) repeated cycles at an increasing strain (@ 50\%) with a fixed strain rate on a virgin sample; Different arrows with colors indicate increasing loading-unloading paths.

as frequently suggested in the literature [15]. Fig. 3(a) depicts data of a single sample stretched up to $375 \%$ deformation with five repeated cycles. It is clear from the figure that during the loading path, the stress-strain behaviour of the material follows a typical nonlinear curve when there is no mechanical pre-treatment applied on the sample. At the beginning, the stiffness of the material increases dramatically and then stabilises quickly on the loading path. From approximately $300 \%$ strain, the stress-strain curve shows significant stiffening. In contrast to the first loading path, the trend in the subsequent cycles experiences a significant stress reduction for the same applied strain, and exhibit a stronger nonlinearity with an S-shaped curve. Note that most of the stress reductions occur in the first two cycles. Moreover, after the third cycle, the differences between the previous cycles and an actual cycle reduce, which result in a stable loading-unloading loop.

We further quantify the existence of the Mullins effect with the help of cyclic tensile tests with increasing strains in subsequent loading cycles. If we apply a strain which is greater than the maximum strain already applied, the stress-strain curve goes along the same loading path of the first cycle, cf. Fig. 3(b). Such a curve represents that the material has significant Mullins effects $[15,41]$. Hence, after performing two different types of tests, we reach a conclusion that although EUP40 is an unfilled elastomer, it shows a significant stress-softening or Mullins effect. Therefore, before performing any viscoelastic experiments, three cycles of pre-treatments have been completed on each virgin sample. The following day, all samples are used for actual viscoelastic experiments. Note that we compare the results of a virgin sample after three to five cycles with the results of the first cycle of the sample after one day rest. They do coincide each other as the polymer do not show any significant self-recovery. However, some polymers show significant self-recovery after Mullins stress-softening tests. For instance, very recently, an extensive study is carried out by our group to demonstrate the self-recovery of a popular silicone rubber after the Mullins stress-softening tests, see Liao et al. [29, 30]. 


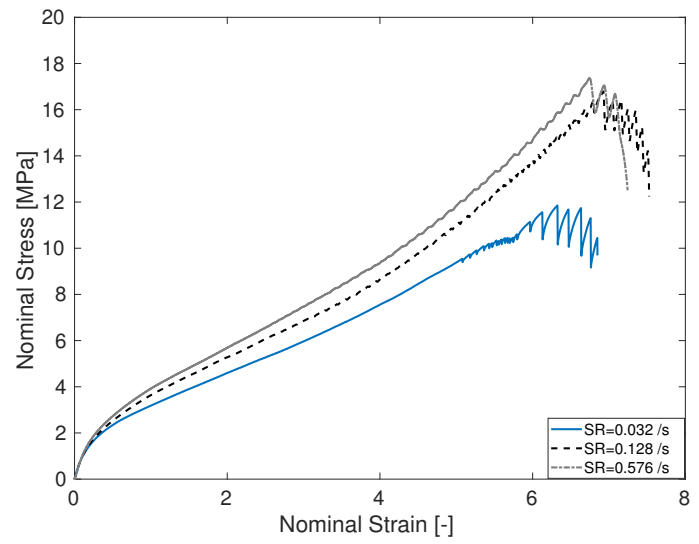

(a)

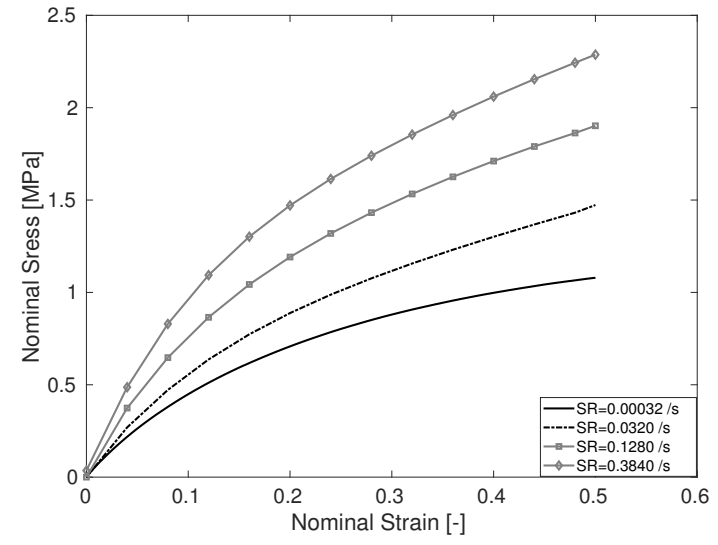

(b)

Figure 4. Stretchability tests : (a) Maximum elongation at break of the EPU40 is determined by performing tests with strain rates (SR) of $0.032 / \mathrm{s}, 0.128 / \mathrm{s}$, and $0.384 / \mathrm{s}$; (b) for quantifying quasi-static stress-strain behaviour, a slow strain rate, 0.00032 /s is added to other three strain rates; Results up to $50 \%$ strain are shown here.

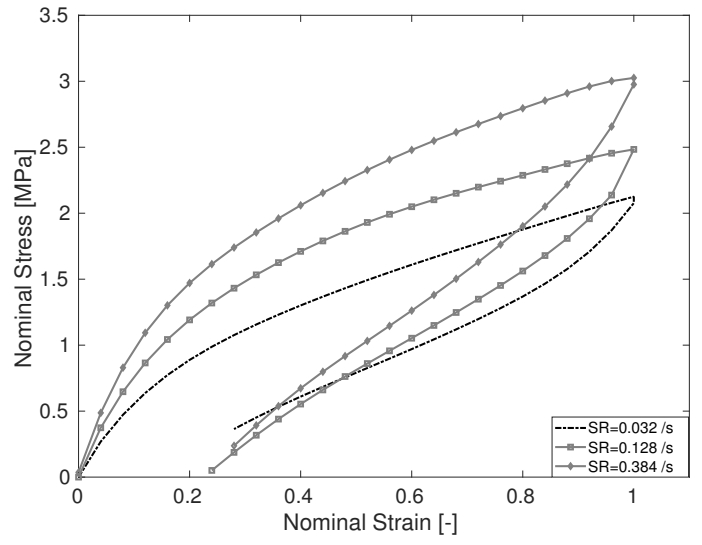

(a)

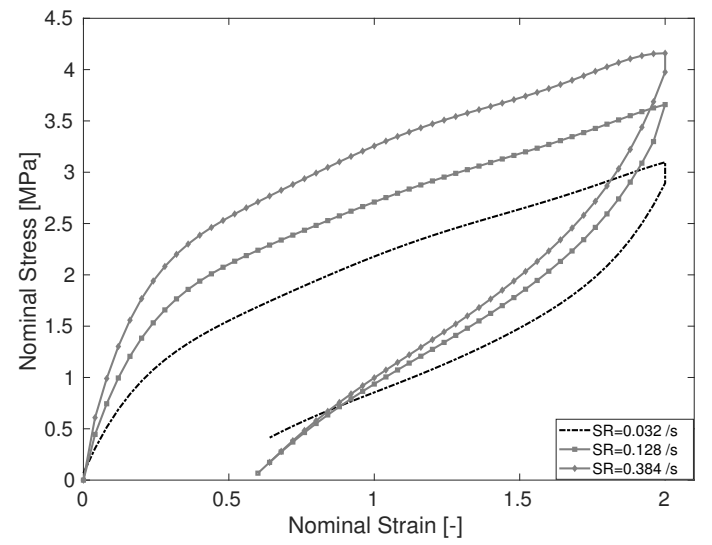

(b)

Figure 5. Cyclic tests under three strain rates, 0.032 /s, 0.128 /s, and 0.384 /s : (a) 100\% strain; (b) $200 \%$ strain. EPU40 shows significant strain-rate sensitivity evidenced by increased nominal stress with increased strain rates. 


\subsection{Stretchability and quasi-static behaviour}

According to a manual supplied by Carbon3D [6], the ultimate elongation at break of the EPU40 is around $275 \%$ (any specific strain rate is not mentioned in the manual), tear strength $23 \mathrm{kN} / \mathrm{m}$, Shore hardness 68 A, and the glass transition temperature $\left(T_{g}\right)$ is $8^{\circ} \mathrm{C}$. However, in order to confirm the stretchability, i.e., the ultimate elongation at break of EUP40 within our lab set-up, strain rates of $0.032 / \mathrm{s}, 0.128 / \mathrm{s}$, and $0.576 / \mathrm{s}$ are selected, which are corresponded to the machine speeds of $50 \mathrm{~mm} / \mathrm{min}, 200 \mathrm{~mm} / \mathrm{min}$, and $900 \mathrm{~mm} / \mathrm{min}$, respectively. All of these strain rates can be considered as low strain rates [27]. Samples are elongated until failure and results are presented in Fig. 4(a). The figure clearly demonstrates that the polymer shows a strong strain rate-sensitivity whereby similar to most other polymers, a larger strain rate shows a higher stress and the vice-versa $[18,28]$. These are representative test results of five samples in which the EPU40 fails at strains ranging between $600 \%$ and $700 \%$ depending on the strain rates. These elongations at break are much more higher than the one mentioned in the Carbon3D manual (275\% strain). Printing conditions such as UV light intensity, printing-induced anisotropy to the bulk samples, properties of the oxygen-permeable layer in the Carbon M2 printer etc, could be responsible for such a large elongation at break. Hence, there is plan to conduct a full-scale printing parameters study in a forthcoming condition. In this case, microscopic study using scanning electron microscope (SEM) needs to be performed in the future to reveal any printinginduced anisotropy in the samples. In summary, it can be concluded that within the printing conditions of the current study, the ultimate strengths at failure of the polymer are varied from $12 \mathrm{MPa}$ to $17 \mathrm{MPa}$ of stretch ratios of 7.0 to 8.0, respectively. In contrast to most other polymers [27, 28], in the case of EPU40, a high strain rate results in a larger elongation at break and the vice-versa. Note that some 3D printed soft polymers, such as PolyJet printed Tangoflex-Verowhite, show reduced elongation at break with slower strain rates, see [31, 54]. Most interesting observation in our experiments is that the stretchability is almost double compared to what is described in the Carbon3D manual [6]. Printing conditions might be responsible for these enhanced elongations at break. Therefore, as soon as Carbon3D printer will be available, there is a plan to conduct extensive investigations to see how printing parameters, such as light intensity, layer thickness, post-curing treatments etc, can affect the stretchability and other mechanical properties of EPU40. Note that the 3D printed EPU40 shows significantly different behaviour than room temperature-cured or elevated temperature-cured elastomeric polyurethanes. For instance, Johlitz et al. [23] conducted extensive investigations on a commercially available elevated temperature-cured $\left(90^{\circ} \mathrm{C}\right.$ for four hours) EPU (Bayer AG, Germany) that has a glass transition temperature of $0^{\circ} \mathrm{C}$. Moreover, if one compares the experimental results of Johlitz et al. [23] with the current study, it is clear that the 3D printed EPU40 shows extreme nonlinear stress-strain behaviour even at $50 \%$ strain.

Our aim, in the following sections, is not only to identify rate-dependence of the polymer but also to find the time-insensitive equilibrium stress-strain relationship. Among other techniques for identifying the equilibrium stress-strain responses, few tests need to be performed at the slowest possible strain rates. Such experiments are called quasi-static tests. For this, samples are stretched at a strain rate of $0.00032 / \mathrm{s}$. This strain rate is significantly slower than the rates applied in the case of stretchability tests in the previous section. Note that we are unable to conduct tests slower than this strain rate. Due to stretching for a very long time, samples are glued to the grippers and the machine is not responding properly. Therefore, we present results up to $50 \%$ strain at a strain rate of 0.00032 /s, see Fig. 4(b). Although the strain rate 0.00032 /s (solid line) is hundred times slower than 0.032 /s, the stress difference is almost the same as in two others strain rates which are only four times (i.e., $0.128 / \mathrm{s}$ ) and twelve times (i.e., $0.384 / \mathrm{s}$ ) higher than 0.032 /s, respectively. It can easily be predicted that any loading-unloading cyclic test, performed at strain rates slower than 0.00032 /s, will not produce stress responses far less than this one. Hence, it is presumed from Fig. 4(b) 


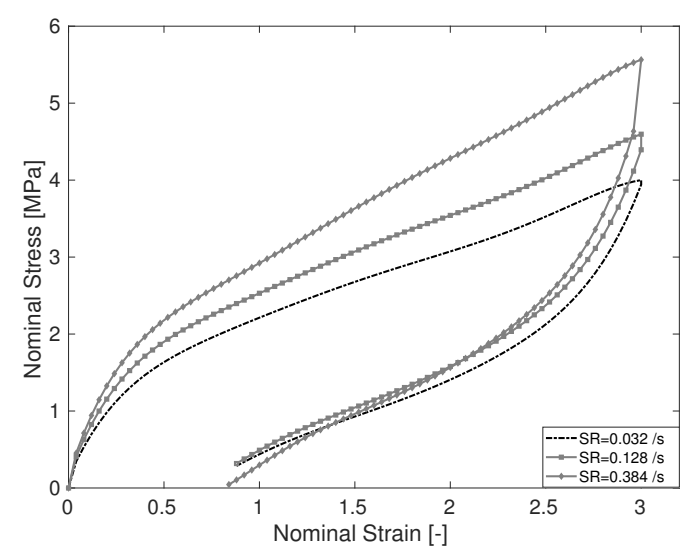

(a)

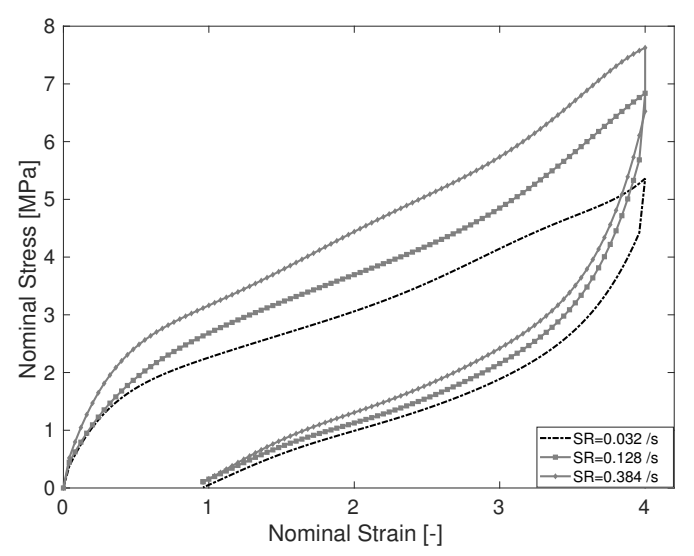

(b)

Figure 6. Loading-unloading cyclic tests at three different strain rates, 0.032 /s, 0.128 /s, and 0.384 /s: (a) 300\% strain; (b) 400\% strain. The polymer shows a significant strain hardening with increasing strains resulting in pronounced nonlinear stress-strain behaviour.

that the results produced by the slowest strain rate $(0.00032 / \mathrm{s})$ are very close to the so-called quasi-static stress states. Furthermore, in order to extract equilibrium stresses at higher strains and to verify the results obtained by the quasi-static tests in this section, asymptotically relaxed stresses from the two relaxation tests will be compared in the following sections.

\subsection{Cyclic experiments}

In an effort to understand the strain rate-dependent behaviour of EUP40, a set of tests are performed at selected strains and strain rates. In this contribution, strain rates of $0.032 / \mathrm{s}, 0.128 / \mathrm{s}$, and $0.384 / \mathrm{s}$ are selected which correspond to the machine velocities, $50 \mathrm{~mm} / \mathrm{min}, 200 \mathrm{~mm} / \mathrm{min}$, and $600 \mathrm{~mm} / \mathrm{min}$, respectively. To demonstrate the stress-strain relationship, four different strains, i.e., 100\%, 200\%, 300\%, and $400 \%$ are selected, see Fig. 5 and Fig. 6. Fig. 5(a) shows results for three different strain rates at $100 \%$ strain, from which it is clear that within the range of strain-rates $(0.032 / \mathrm{s}-0.384 / \mathrm{s})$, EPU40 demonstrates significant strain rate-sensitivity. Moreover, for the polymer, a higher strain rate yields a larger magnitude of the stress and the vice-versa. Within the hundred percent strain, the loading-unloading curves show significant nonlinearity whereby a steep strain hardening occurs within the range of the first twenty percent strain. It is noteworthy that, according to a manual supplied by Carbon3D [6], EPU40 has a stress of $3.0 \mathrm{MPa}$ at 100\% strain with a machine speed of $500 \mathrm{~mm} / \mathrm{min}$. A similar stress value (3.1 MPa at $100 \%$ strain with a machine speed of $600 \mathrm{~mm} / \mathrm{min} \approx 0.384 / \mathrm{s}$ strain rate) is obtained in our study, see the top loading-unloading curve of Fig. 5(a). Such a close correspondence between the current experimental findings and the Carbon3D manual establishes the fact that samples produced and used in the study match the original standard of CarbonM2 printer conditions and confirm accuracy of our experimental results.

Fig. 5(b) compares the loading-unloading curves for the three different strain rates at $200 \%$ strain, from which it is clear that a higher strain yields larger dissipations that can be measured by the areas bounded between the loading and the unloading curves. Similarly, cyclic stress-strain results for $300 \%$ and $400 \%$ strains are presented in Fig. 6, from which significant nonlinearities in stress-strain curves and initial strain harden- 


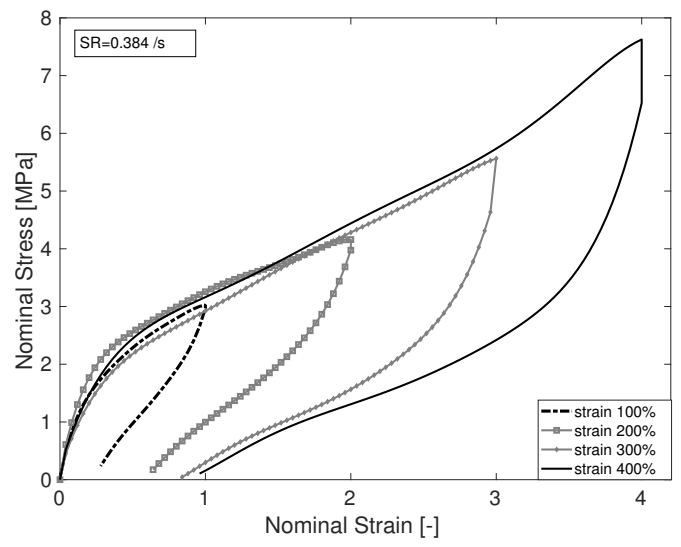

Figure 7. Loading-unloading cyclic tests at a fixed strain rate of $0.384 / \mathrm{s}$ up to $400 \%$ strain. It is clear from the figure that with the increase of strain, the stress-strain curve becomes highly nonlinear with an upturn, a typical stress-strain behaviour of elastomeric polymers

ing within the first twenty percent strain of the polymer have clearly been observed. Moreover, in contrast to strains below $300 \%$, the stress-strain response at $400 \%$ strain depicts an upturn tendency of the curves, which is very similar to the so-called ' $S$ ' curve (hardening-softening-hardening) of stress-strain relations, typical for classical rubber-like polymers $[13,51]$. Note that upon unloading, none of the sample returns to its original position as shown in Fig. 5 and Fig. 6. One of the key findings of these loading-unloading curves is that the dissipative loops as well as their nonlinearities not only depend on the strains but also on the strain rates. Several studies described such behaviour as the result of the so-called nonlinear viscosity. Amin et al. $[2,3]$ experimentally observed nonlinear viscosities in natural and high damping rubbers whereas Koprowski et al. $[25,26]$ investigated the same phenomenon in ethylene propylene diene monomer (EPDM) and cellular rubbers. A close inspection of all figures presented in this section for various strains and strain rates clearly reveals that a higher strain rate results in a larger residual strain and the vice-versa. Similarly, a higher deformation yields a greater residual strain, see Fig. 7. It is noteworthy to mention that many soft polymers show a significant recovery of the residual strain if samples are kept in a stress-free condition for a couple of hours [30]. However, in the current study, we do not conduct any stress and strain recovery study. This is deferred for future study of the EPU40. In summary, we can conclude that EUP40 shows all major characteristics typical for a viscoelastic polymer.

\subsection{Relaxation behaviour : Single-step experiments}

Stress relaxation is an important property for soft viscoelastic materials. Single-step relaxation tests will simply help us to understand the equilibrium stress response of polymers. In order to perform relaxation experiments, every pre-conditioned sample is stretched up to the desired strain and allowed relaxation. Since no previous viscoelastic study on EPU40 is available, at first, the task is to identify the required time for the stress relaxation. To this end, a sample is stretched up to the desired level and the stress evolution over time is monitored for several hours, see Fig. 8(b) in which the nominal stress is plotted over the relaxation time. It is clear from Fig. 8(b) that most of the so-called overstress or the non-equilibrium stress is relaxed within first few minutes. As the holding time increases, the actual stress reaches to a relaxed level which does not change significantly. Note, however, that within thirty minutes of the relaxation, more than $95 \%$ of the overstress is disappeared. Hence, rest of the relaxation tests are performed with half an hour of relaxation 


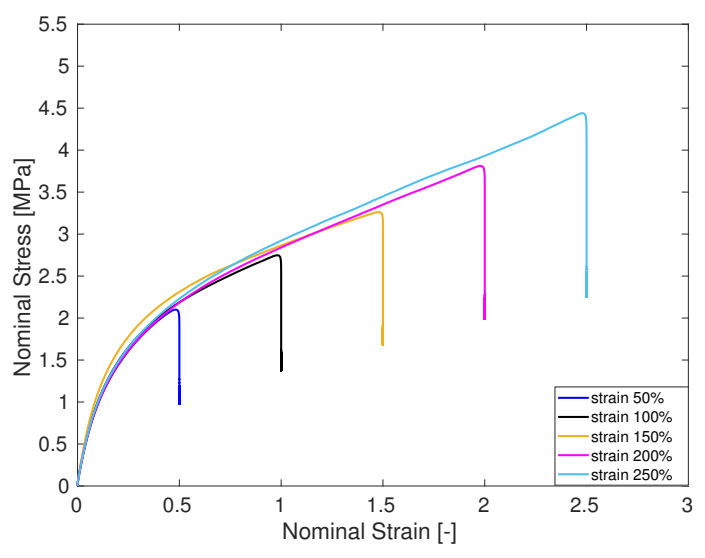

(a)

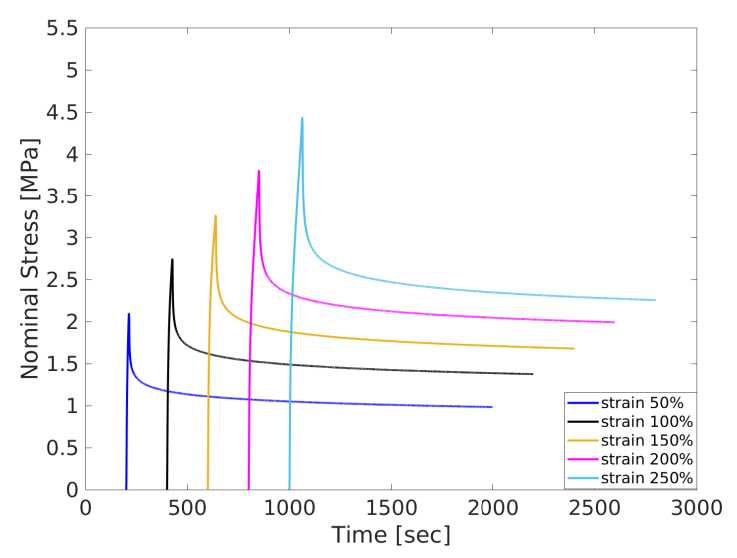

(b)

Figure 8. Single-step relaxation tests performed at 50\%, 100\%, 150\%, 200\%, and $250 \%$ strains. (a) Nominal stress over nominal strain, (b) stress relaxation over time (sec) where, for a better illustration, the stress histories obtained from two different strain relaxation tests have been separated from each other by $200 \mathrm{~s}$.

time. The same relaxation time is allocated in each step for the multi-step relaxation tests.

With half an hour relaxation time, several relaxation tests for 50\%, 100\%, 150\%, 200\%, and 250\% strains are conducted. Relevant findings are presented in Fig. 8, in which the total nominal stresses are plotted against nominal strain in Fig. 8(a), while the nominal stress time evolution is plotted in Fig. 8(b). For a clearer illustration purpose, the stress over relaxation time curves are moved, see Fig. 8(b). When the stress is relaxed after half an hour holding time, it is taken as the equilibrium stress. This stress is normally time-independent, i.e., it does not associated with any polymeric viscosity. Some more information can be revealed from Fig. 8. For example, if a sample is stretched at a higher strain, its relaxed stress will be achieved at a longer time. In other words, the relaxation time increases as the strain level is increased, see Fig. 8(b). We have calculated relaxed equilibrium stresses for several strain levels. These will help us in obtaining the basic elastic curve of the polymer. These equilibrium stresses are essential to identify the material constants in the hyperelastic law presented in Sec. 4.

\subsection{Relaxation behaviour: Multi-step experiments}

Some devices made of soft polymeric materials can operate with strains at various levels. In such cases, materials will experience stress relaxation at multiple times. Hence, it is paramount to assess materials' stress relaxation at multiple strains [11]. In addition, among several ways, the identification of the equilibrium stress of a polymeric material can be obtained by performing multi-step relaxation tests. In the case of step-wise relaxation tests, we deform a sample up to a pre-specified strain and then it is allowed to relax for half an hour period. In the multi-step tests, the specimen is further stretched to the next level instead of unloading it. In this case, we can extract several equilibrium stresses by performing the test on a single sample. In our study, a step-wise test is carried out at an interval of 50\% strain until $400 \%$. Fig. 9 shows the loading curves in terms of nominal stress versus relaxation time. 


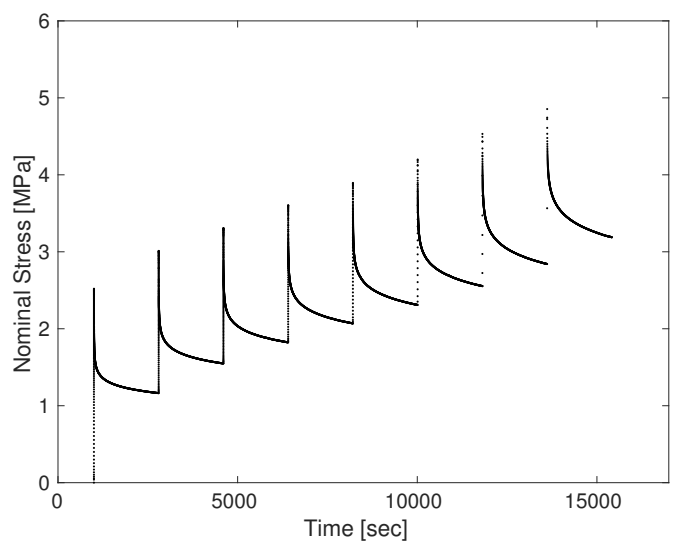

Figure 9. Multi-step relaxation tests at 50\%, 100\%, 150\%, 200\%, 250\%, 300\%, 350\%, and 400\% strains. Nominal stress is plotted against relaxation time.

\section{Constitutive modelling of finite strain viscoelasticity}

The starting point for modelling a rubber-like material is the so-called strain energy function $\Psi(\boldsymbol{C})$, where $\boldsymbol{C}$ is the right Cauchy-Green strain tensor that relates to the deformation gradient $\boldsymbol{F}$ via $\boldsymbol{C}=\boldsymbol{F}^{T} \boldsymbol{F}$. In order to capture the time-dependent behaviour of polymers, it is frequently regarded that the strain energy function depends further on internal variable(s) $\boldsymbol{A}$, i.e., $\Psi(\boldsymbol{C}, \boldsymbol{A})$. Rubber-like materials are widely considered as incompressible materials where the energy function can be expressed as

$$
\Psi(\boldsymbol{C}, \boldsymbol{A})=-p(J-1)+\tilde{\Psi}^{e}(\boldsymbol{C})+\sum_{j=1}^{N} \tilde{\Psi}^{v, j}\left(\boldsymbol{C}, \boldsymbol{A}_{j}\right)
$$

where, $N$ denotes the number of elements, $J=\operatorname{det} \boldsymbol{F}$, and $p$ is a Lagrange multiplier [21, 24]. For this, the second Piola-Kirchhoff stress becomes

$$
\boldsymbol{S}=-p \boldsymbol{C}^{-1}+2 \frac{\partial \Psi^{e}}{\partial \boldsymbol{C}}+2 \sum_{j=1}^{N} \frac{\partial \Psi^{v, j}}{\partial \boldsymbol{C}} .
$$

For capturing the time-dependent behaviour of polymers, we require two separate energy functions in order to have a complete representation of the constitutive model in Eqn (1). As EPU40 shows a significant nonlinearity in its stress-strain relationship, cf. Sec. 3.3, a more complex strain energy function needs to be chosen. A three-parameter Carroll [7] strain energy function is selected here thanks to its outstanding predictability in capturing hyperelastic experimental data for a wide range of polymeric materials, see Steinmann et al. [51], Hossain et al. [19]. The three-parameter Carroll energy function is given as follows

$$
\Psi^{e}(\boldsymbol{C})=a I_{1}+b I_{1}^{4}+c \sqrt{I_{2}}
$$

where, $a, b, c$ are called fitting constants, while $I_{1}, I_{2}$ are the first and second invariants, respectively, of $C$. With the definition of stress in Eqn (2) and using the relation in (3), the elastic stress tensor can be calculated: 


$$
\boldsymbol{S}^{e}=2 \frac{\partial \Psi^{e}}{\partial \boldsymbol{C}}=\left[2 a+8 b I_{1}^{3}\right] \boldsymbol{I}+c\left[I_{1} \boldsymbol{I}-\boldsymbol{C}\right] I_{2}^{-1 / 2}, \quad \boldsymbol{I} \equiv \delta_{i j}
$$

The viscous strain energy $\tilde{\Psi}(\boldsymbol{C}, \boldsymbol{A})$ remains to be specified along with an evolution law for tracking $\boldsymbol{A}$. For these, an approach recently proposed by Liao et al. [28] is adopted, which is mainly based on the earlier works of Sedlan [50], Koprowski et al. [25, 26], Scheffer et al. [47]. We, furthermore, replace the internal variable $\boldsymbol{A}$ with $\boldsymbol{C}_{v}$, where $\boldsymbol{C}_{v}$ is related to the viscous part of the elastic-viscous decomposition of the deformation gradient, i.e., $\boldsymbol{F}=\left\{\boldsymbol{F}_{e}^{j} \boldsymbol{F}_{v}^{j}\right\}_{j=1}^{N}$, see Haldar and Pal [22], Perić and Dettmer [43], Mehnert et al. [37]. The multiplicative decomposition of the deformation gradient results in the following expressions $\boldsymbol{C}_{e}^{j}=\left[\boldsymbol{F}_{e}^{j}\right]^{T} \boldsymbol{F}_{e}^{j}$ and $\boldsymbol{C}_{v}^{j}=\left[\boldsymbol{F}_{v}^{j}\right]^{T} \boldsymbol{F}_{v}^{j}$. In an effort order to predict the complex experimental data demonstrated in Sec. 2, we take two different energy functions in the viscous regime. The first part of the energy function is a higher order Yeoh model while the second part follows a Neo-Hooke model as

$$
\Psi^{v}\left(\boldsymbol{C}_{e}\right)=\sum_{j=1}^{s} \Psi_{Y}^{v, j}+\sum_{j=s+1}^{N} \Psi_{H}^{v, j}=\sum_{j=1}^{s} c_{4}^{j}\left[I_{1 e}^{j}-3\right]^{3}+\sum_{j=s+1}^{N} c_{5}^{j}\left[I_{1 e}^{j}-3\right]
$$

Note that two energy functions are employed to describe the viscous part, cf. Eqn (5), which, according to Eqn (7), result in two separate evolution equations. For the Yeoh-type viscous strain energy $(5)_{1}$, the evolution equation defined in Eqn (7) becomes

$$
\dot{\boldsymbol{C}}_{v}^{j}=\frac{12}{\tilde{\tau}_{1}^{j}(\boldsymbol{C}, \boldsymbol{D})}\left[I_{1 e}^{j}-3\right]^{2}\left[\boldsymbol{C}-\frac{1}{3}\left[\boldsymbol{C}:\left[\boldsymbol{C}_{v}^{j}\right]^{-1}\right] \boldsymbol{C}_{v}^{j}\right], \quad j=1, \cdots, s ; \quad \tilde{\tau}_{1}^{j}(\boldsymbol{C}, \boldsymbol{D})=\frac{\tilde{\eta}^{j}(\boldsymbol{C}, \boldsymbol{D})}{c_{4}^{j}}
$$


while the Neo-Hooke viscous function $(5)_{2}$ gives another evolution law as

$$
\dot{\boldsymbol{C}}_{v}^{j}=\frac{4}{\tilde{\tau}_{2}^{j}(\boldsymbol{C}, \boldsymbol{D})}\left[\boldsymbol{C}-\frac{1}{3}\left[\boldsymbol{C}:\left[\boldsymbol{C}_{v}^{j}\right]^{-1}\right] \boldsymbol{C}_{v}^{j}\right], \quad j=(s+1), \cdots, N ; \quad \tilde{\tau}_{2}^{j}(\boldsymbol{C}, \boldsymbol{D})=\frac{\tilde{\eta}^{j}(\boldsymbol{C}, \boldsymbol{D})}{c_{5}^{j}}
$$

\subsection{Scalar forms of the viscoelastic model}

In Sec. 2, we conduct various tests following standards of an uniaxial model of deformation. Therefore, all relevant equations of the constitutive law will be calculated as a set of one-dimensional equations. For uniaxial tests, samples are stretched in one directions and other directions are free to move. Note that $\left\{\lambda_{i}\right\}_{i=1}^{3}$ are the square roots of the principal values of the right-Green strain tensor $C$. Owing to the incompressibility condition for rubber-like materials, the determinant of the deformation gradient results in $\lambda_{1} \lambda_{2} \lambda_{3}=1$. As a results of these information, Eqn (4) becomes

$$
P^{e}=\left[2 a+8 b\left[2 \lambda^{-1}+\lambda^{2}\right]^{3}+c\left[1+2 \lambda^{3}\right]^{-\frac{1}{2}}\right]\left[\lambda-\lambda^{-2}\right] .
$$

For detailed derivations, we refer readers to Hossain [19], Steinmann et al. [51]. The viscous stress from Eqn (6) is

$$
\begin{aligned}
P^{v} & =\sum_{j=1}^{s} P^{v, j}+\sum_{j=s+1}^{N} P^{v, j} \\
& =\sum_{j=1}^{s} 6 c_{4}^{j}\left[\frac{\lambda^{2}}{\left[\lambda_{v}^{j}\right]^{2}}+\frac{2 \lambda_{v}^{j}}{\lambda}-3\right]^{2}\left[\frac{\lambda}{\left[\lambda_{v}^{j}\right]^{2}}-\frac{\lambda_{v}^{j}}{\lambda^{2}}\right]+\sum_{j=s+1}^{N} 2 c_{5}^{j}\left[\frac{\lambda}{\left[\lambda_{v}^{j}\right]^{2}}-\frac{\lambda_{v}^{j}}{\lambda^{2}}\right] .
\end{aligned}
$$

Note that $\lambda_{v}^{2}$ is the eigenvalue of $\boldsymbol{C}_{v}$. An one-dimensional form of the first evolution law (8) is calculated as

$$
\dot{\overline{\lambda_{v}^{j}}}=\frac{4}{\tilde{\tau}_{1}^{j}(\lambda, \dot{\lambda})}\left[\frac{\lambda^{2}}{\left[\lambda_{v}^{j}\right]^{2}}+\frac{2 \lambda_{v}^{j}}{\lambda}-3\right]^{2}\left[\frac{\lambda^{2}}{\lambda_{v}^{j}}-\frac{\left[\lambda_{v}^{j}\right]^{2}}{\lambda}\right], \quad j=1, \cdots, s
$$

and the same for the second evolution equation (9) is

$$
\dot{\overline{\lambda_{v}^{j}}}=\frac{4}{3 \tilde{\tau}_{2}^{j}(\lambda, \dot{\lambda})}\left[\frac{\lambda^{2}}{\lambda_{v}^{j}}-\frac{\left[\lambda_{v}^{j}\right]^{2}}{\lambda}\right], \quad j=(s+1), \cdots, N .
$$

Note that expressions for the relaxation functions $\tilde{\tau}_{1}^{j}(\lambda, \dot{\lambda})$ and $\tilde{\tau}_{2}^{j}(\lambda, \dot{\lambda})$ will be determined with the help of experimental data from Section 5. Both the aforementioned scalar-valued differential equations for the internal variables $\lambda_{v}^{j}$ require an integration scheme. Using implicit Euler-backward integration scheme, Eqn (13) becomes

$$
\begin{aligned}
\lambda_{v}^{j} & =\lambda_{v}^{j, n}+\frac{4 \Delta t}{3 \tilde{\tau}_{2}^{j}(\lambda, \dot{\lambda})}\left[\frac{\lambda^{2}}{\lambda_{v}^{j}}-\frac{\left[\lambda_{v}^{j}\right]^{2}}{\lambda}\right], \\
f\left(\lambda_{v}^{j}\right) & =\lambda_{v}^{j}-\lambda_{v}^{n, j}-\frac{4 \Delta t}{3 \tilde{\tau}_{2}^{j}(\lambda, \dot{\lambda})}\left[\frac{\lambda^{2}}{\lambda_{v}^{j}}-\frac{\left[\lambda_{v}^{j}\right]^{2}}{\lambda}\right]
\end{aligned}
$$




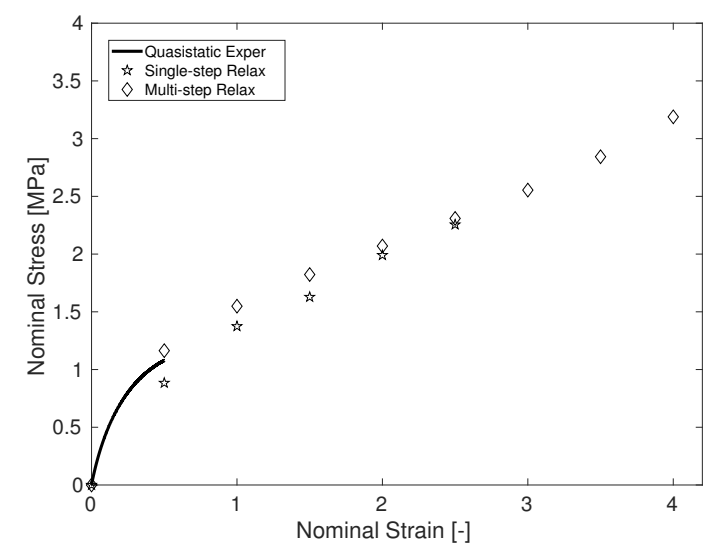

(a)

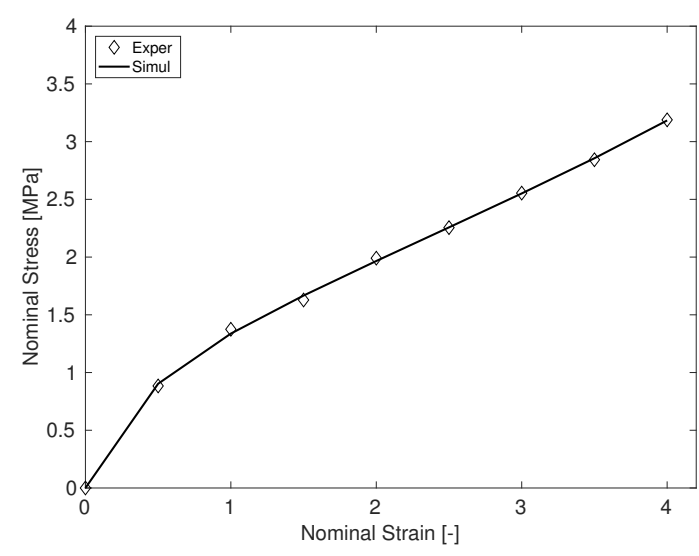

(b)

Figure 10. Elastic parameters identification: (a) Equilibrium stress data extracted from three different types of experiments,(b) equilibrium data from multi-step relaxation test is fitted to Carroll hyperelastic model.

390

where, $[\bullet]^{k}=[\bullet]\left(t_{k}\right)$ and $\Delta t=t_{n+1}-t_{n}$.

\section{Model validation followed by parameters identification}

In this section, at first all materials constants as in Eqns (10), (11), (12), and (13) will be identified using the data presented in Sec. 2. For the identification purpose, we use a built-in function lsqcurvefit from the mathematical package Mat lab.

\subsection{Elastic parameter identification}

For the hyperelastic constitutive equation (10), the first step is to separate the relaxed (equilibrium) stress data. Afterwards, these data are to be used in identifying the elastic parameters only, see [2, 23]. The hyperelastic function characterized by three elastic parameters, $a, b$, and $c$ as per Eqn (10) is due to the Carroll model. In order to obtain the time-independent elastic data, several techniques have been used, see $[2,18]$.

In this study, three most widely used methodologies for identifying the basic elasticity are explored. The first option is to extract equilibrium stress data from the relaxation tests. After the end of half an hour holding time, we obtain asymptotical plateau for each strain, see Fig. 8(b). The data from the relaxation tests of 50\%, 100\%, 150\%, 200\%, and 250\% strains are extracted. A similar but less costly approach (in terms of samples used) is the multi-step relaxation tests data, see Fig. 9. The asymptotic stresses reached after each step-wise strain from the multi-step relaxation tests are selected. The last option applied in the current study is the quasi-static experimental data from monotonic tests in Fig. 4(b). For that, the strain rate of $0.00032 / \mathrm{s}$ is chosen while tests are performed for strains of up to 50\%. The equilibrium stresses obtained from all the three different techniques are presented in Fig. 10(a), from which it is clear that the three sets of data are following the same trend. Initially, the equilibrium data from the single-step tests are comparatively lower than the multi-step tests data and the quasi-static data fit in-between the two extreme cases. Moreover, the differences between the two sets of equilibrium data reduce significantly at the higher strains. We have a complete set of data up to $400 \%$ strain from the step-wise tests that demonstrate a 


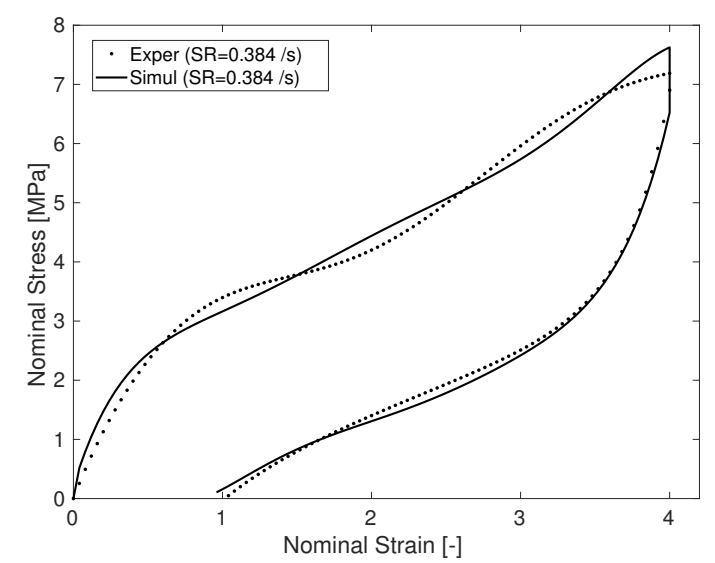

(a)

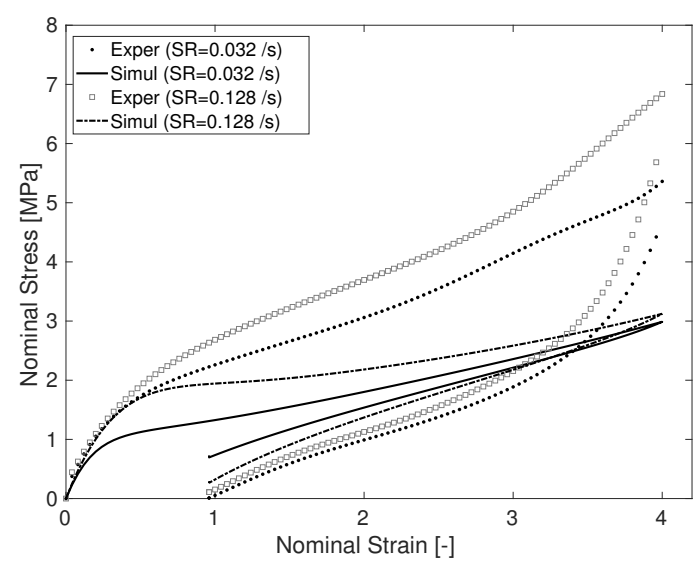

(b)

Figure 11. Viscous parameters identification with a loading-unloading cyclic data for $400 \%$ strain at a strain rate of 0.384 /s with linear relaxation times: (a) Model fitting with one Yeoh and four Neo-Hooke Maxwell elements with ten viscous parameters (total thirteen parameters; three elastic and ten viscous), (b) model validation with one Yeoh and four Neo-Hooke Maxwell elements (thirteen parameters in total).

strong nonlinear stress-strain relationship. Hence, the end points of each strain step from the multi-step relaxation test are chosen to obtain elastic data up to $400 \%$ strain. The fitting of the elastic part of the model is presented in Fig. 10(b) using lsqcurvefit function of Matlab. The identified elastic parameters are $[a, b, c]=[2.868 \mathrm{e}-01,1.4183 \mathrm{e}-07,7.846 \mathrm{e}-01] \mathrm{MPa}$. Note that the Carroll model perfectly captures the equilibrium stress data up to $400 \%$ strain.

\subsection{Viscous parameter identification: Linear viscosity}

Three hyperelastic parameters $a, b, c$ appearing in Eqn (10) are already identified in Sec. 5.1. Now, we have to identify the viscous constants, namely the overstress moduli $\left\{c_{4}^{j}, c_{5}^{j}\right\}_{j=1}^{N}$ and the relaxation times $\left\{\tau_{1}^{j}, \tau_{2}^{j}\right\}_{j=1}^{N}$. At first, it is assumed that the relaxation times, which are directly related to the material viscosities (see Eqns (8) and (9)), are not changing with respect to applied strains or strain rates, i.e. these are constant parameters appearing in Eqns (11), (12), and (13). In search of the relaxation constants, the cyclic data for $400 \%$ strain are selected with only one strain rate, i.e., 0.384 /s. First, it is necessary to specify the quantity of the dash-pot elements that can capture the strong nonlinear stress-strain data observed at these strain and strain rate, see Fig. 6(b). From the parameter identification and computational viewpoints, a minimum number of material parameters is highly desirable. At first, the data of $400 \%$ with the strain rate of 0.384 /s are fitted with only one Yeoh-type and one Neo Hooke-type Maxwell elements. However, as expected, two Maxwell elements (four parameters) do not capture the strong nonlinearity observed in the stress-strain data. Afterwards, constitutive equations obtained with one Yeoh and four Neo-Hooke Maxwell elements (total ten viscous parameters) are fitted with the data. The results illustrated in Fig. 11(a) clearly indicate that the constitutive relations with the five Maxwell elements containing ten parameters (in addition to the three hyperelastic parameters) can capture well the experimental data including the second strain hardening behaviour in the hardening-softening-hardening curve. Furthermore, the data are fitted with one Yeoh and five Neo-Hooke Maxwell elements and the identification is similar as in Fig. 11(a). Despite the addition of one extra Neo-Hooke Maxwell element that yields two more viscous parameters, the results do not differ significantly in comparison to one Yeoh and four Neo-Hooke type Maxwell elements. Note that 


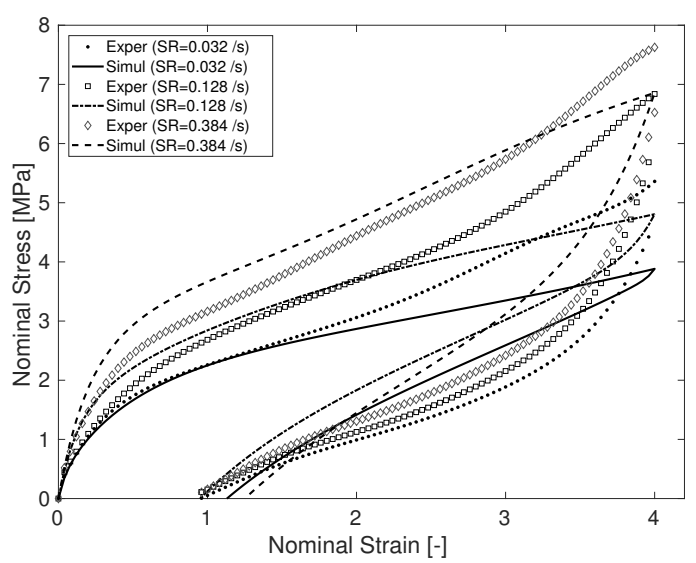

(a)

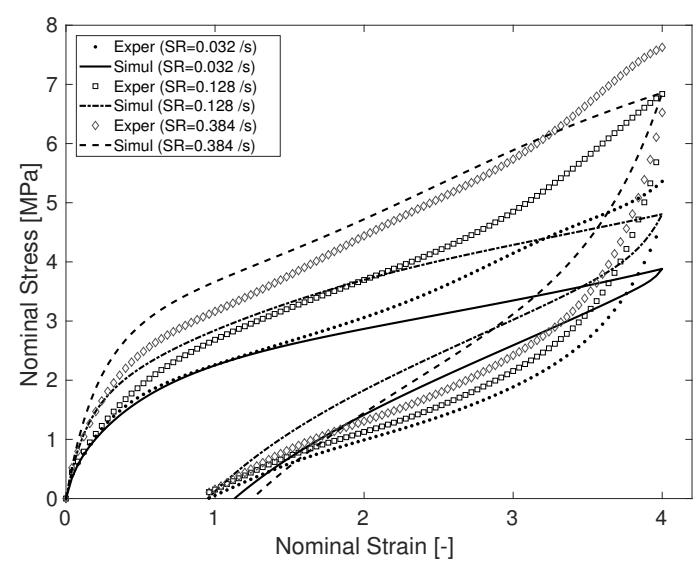

(b)

Figure 12. Viscous parameters identification with a loading-unloading cyclic data of $400 \%$ strain at strain rates of $0.384 / \mathrm{s}, 0.128$ /s, and 0.032 /s: (a) Model fitting with one Yeoh and four Neo-Hooke Maxwell elements, (b) model fitting with one Yeoh and five Neo-Hooke Maxwell elements with twelve viscous parameters (total fifteen parameters; three elastic and twelve viscous). The increase of the Maxwell elements higher than five does not enhance the model fitting quality with respect to experimental data

the hyperelastic constants $a, b$, and $c$ are pre-identified in the case of viscous parameters identification.

As per the definition of model validation, the constitutive equations must predict a separate set of new data. The validation of $400 \%$ data with two other strain rates, i.e., $0.128 / \mathrm{s}$ and $0.032 / \mathrm{s}$ is shown in Fig. 11(b). It is clear from the figure that although the model fits well with one strain rate $(0.384 / \mathrm{s})$, an extremely poor prediction is observed at other strain rates for the same strain $(400 \%)$. By changing only the time increment $(\Delta t)$ in the simulation codes as per in Eqn (14), the model can not capture two other strain rates data. Now, the three strain rates are simultaneously taken into the identification process, which is known as the simultaneous optimization, see [19, 23]. The fitting results are demonstrated in Fig. 12(a) and Fig. 12(b) for five and six Maxwell elements, respectively. The simultaneous optimization technique for five Maxwell elements containing ten viscous parameters largely capture the hardening-softening behaviour of the polymer. However, by applying the technique, neither five nor six Maxwell elements can capture a significant hardening response of the material that is observed above $300 \%$ strains. As discussed in the literature, such extreme nonlinearities of the stress-strain behaviour depend both on strains and strain rates. Hence, linear viscosities, i.e., constant values of the relaxation times do not capture the hardening-softening-hardening behaviour observed in the polymer.

\subsection{Viscous parameters identification: Nonlinear viscosity}

Experimentally obtained stress-strain curves in our study typically show three regions: at first a linear region, then a softening region in the middle and finally a hardening zone at the end. This is the common behaviour of rubber-like materials at very large strains [13]. Hence, a new strategy needs to be devised which will incorporate strain and strain rates-dependent viscosity functions instead of taking these as simple constants. Motivated by Amin et al. [2, 3], Zhou et al. [64], strain and strain rate-dependent functions for the relaxations are formulated as 
Table 1: Elastic and viscous parameters, respectively; $a, b, c, c_{4}^{j}, c_{5}^{j}$ in [MPa] and $\tau_{1 i}^{j}, \tau_{2 i}^{j}, k_{j i}$ in [sec]. Carrol model used in capturing equilibrium data results in three hyperelastic parameters while four Maxwell elements with strain and strain rate-dependent relaxation functions yield thirteen viscous material constants

\begin{tabular}{cccccc}
\hline$a$ & $b$ & $c$ & $c_{5}^{2}$ & $c_{5}^{3}$ & - \\
\hline $2.868 \mathrm{e}-01$ & $1.4183 \mathrm{e}-07$ & $7.846 \mathrm{e}-01$ & $4.464 \mathrm{e}-01$ & $3.382 \mathrm{e}+00$ & - \\
\hline- & - & - & $\tau_{2}^{2}$ & $\tau_{2}^{3}$ & - \\
\hline \hline- & - & - & $7.388 \mathrm{e}+01$ & $4.367 \mathrm{e}-01$ & - \\
\hline$\tau_{1}^{1}$ & $\tau_{11}$ & $\tau_{12}$ & $\tau_{13}$ & $k_{13}$ & $c_{4}^{1}$ \\
\hline- & $2.500 \mathrm{e}+00$ & $4.019 \mathrm{e}-01$ & $3.953 \mathrm{e}+00$ & $4.916 \mathrm{e}+00$ & $8.201 \mathrm{e}-01$ \\
\hline \hline$\tau_{2}^{1}$ & $\tau_{21}$ & $\tau_{22}$ & - & $k_{22}$ & $c_{5}^{1}$ \\
\hline- & $4.35543 \mathrm{e}-12$ & $1.626 \mathrm{e}+02$ & - & $6.618 \mathrm{e}+01$ & $5.792 \mathrm{e}-01$ \\
\hline
\end{tabular}

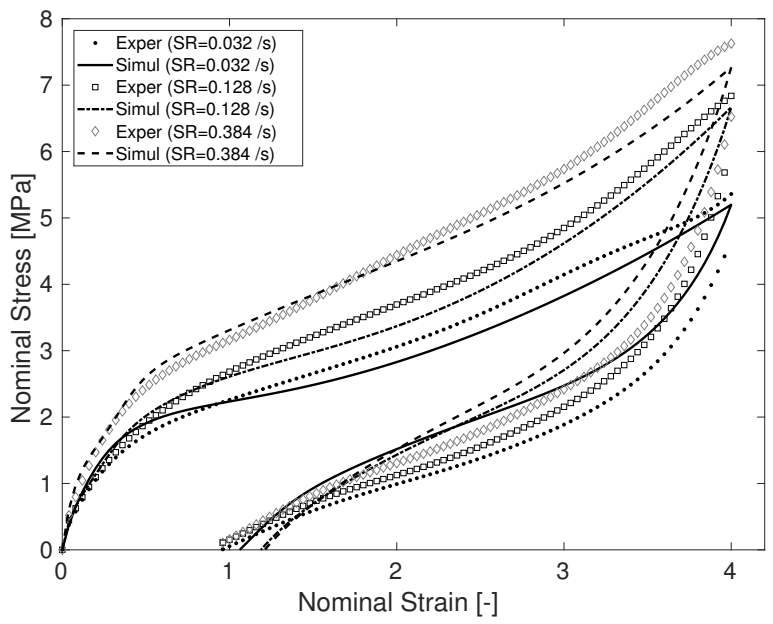

Figure 13. Model parameter identification with loading-unloading cyclic data for $400 \%$ strain with strain rates of $0.384 / \mathrm{s}, 0.128$ /s, and 0.032 /s: By using two strain and strain rate-dependent relaxation functions along with two relaxation constants, the model captures the hardening-softening-hardening behaviour observed in the EPU40 polymer. 
$\tau_{1}^{1}(\boldsymbol{C}, \boldsymbol{D})=\tau_{11}+\left[\tau_{12}+\tau_{13} \exp \left(-k_{13}\|\boldsymbol{D}\|\right)\right][\operatorname{tr} \boldsymbol{C}-3]^{2}, \quad \tau_{2}^{1}(\boldsymbol{C}, \boldsymbol{D})=\tau_{21}+\tau_{22} \exp \left(-k_{22}\|\boldsymbol{D}\|\right)$

which, in the uniaxial tensile case, take the following forms

$$
\tau_{1}^{1}=\tau_{11}+\left[\tau_{12}+\tau_{13} \exp \left(-k_{13} \dot{\lambda} / \lambda\right)\right]\left[\lambda^{2}+2 \lambda^{-1}-3\right]^{2}, \quad \tau_{2}^{1}=\tau_{21}+\tau_{22} \exp \left(-k_{22} \dot{\lambda} / \lambda\right) .
$$

The other remaining relaxation parameters in the evolution equations are kept as constants. Note that the last part with a quadratic function for the strain in Eqn $(15)_{1}$ is due to the strain-dependent nonlinear viscosity. Such a quadratic strain-dependent relaxation function is phenomenologically devised by Amin et al. [2] and Koprowski et al. [25, 26]. Furthermore, Zhou et al. [64] proved that nonlinear viscosity/relaxation functions have micro-mechanical bases. Now, the viscous parameters, namely the overstress moduli $\left\{c_{4}^{j}, c_{5}^{j}\right\}_{j=1}^{N}$ and the relaxation times $\left\{\tau_{1}^{j}, \tau_{2}^{j}\right\}_{j=1}^{N}$ will need to be identified with the newly defined functions in Eqn (15). The loading-unloading cyclic data for $400 \%$ strain with three strain rates, i.e., $0.032 / \mathrm{s}, 0.128 / \mathrm{s}$, and 0.384 $/ s$ are used for the viscous parameters' identification. The question is how many Maxwell elements are required to capture the strong nonlinear stress-strain data observed at this strain and strain rate. Moreover, should all Maxwell elements have strain-and strain rate-dependent viscosity functions? As discussed above, at least four Maxwell elements are required to capture the nonlinearity observed in the data. However, among four elements, nonlinear viscosity/relaxation functions are introduced only for one Yeoh and one Neo Hooke elements and rest of the two relaxation functions are kept as constants. The data for $400 \%$ strain with three strain rates, i.e., $0.032 / \mathrm{s}, 0.128 / \mathrm{s}$, and $0.384 / \mathrm{s}$ are fitted only with one Yeoh-type and three Neo Hooke-type Maxwell elements. The experimental findings are depicted in Fig. 13, from which it can be concluded that constitutive relations with four Maxwell elements with thirteen parameters (in addition to the three hyperelastic parameters) closely capture the experimental data. Owing to the addition of rate-dependent factors in the relaxation functions, the model can accurately capture significant hardeningsoftening-hardening behaviour observed in the EPU40 polymer. Further additions of Maxwell elements do not improve the quality of the model fitting. Note that the hyperelastic parameters $a, b$, and $c$ are kept frozen during the identification of the viscous parameters. At this stage, a validation of the model with all identified parameters is required, see Table 1 for material constants.

\subsection{Constitutive model validation}

Now all viscoelastic parameters are obtained. At this stage, the parameters in Table 1 need to be used to validate the model.

\subsubsection{Validation by strain-rate dependent data}

At first, the loading-unloading cyclic data of various strain rates and strains are used. Model validations with $100 \%, 200 \%$, and 300\% strains at strain rates of 0.032 /s, 0.128 /s, and 0.384 /s are presented in Fig. 14 and Fig. 15, respectively, with total sixteen viscoelastic parameters (three hyperelastic and thirteen viscous). All validation examples show good correlations with the data. In the case of experimental data, where only hardening-softening is observed, the constitutive law is less accurate in predicting experimental data. For instance, excellent predictions for 300\% strain with all three strain rates are observed. However, for $100 \%$ or lower strains and for low strain rates such as $0.032 / \mathrm{s}$ and $0.128 / \mathrm{s}$, the model results in less accurate agreement with experimental data. Further fine tuning of the strain and strain rate-dependent relaxation functions with more advanced optimization algorithms for the parameters' identification might improve the 


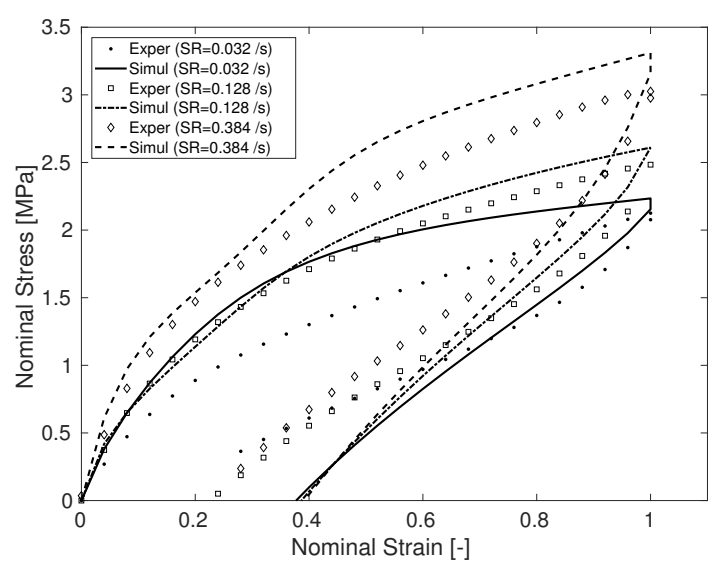

(a)

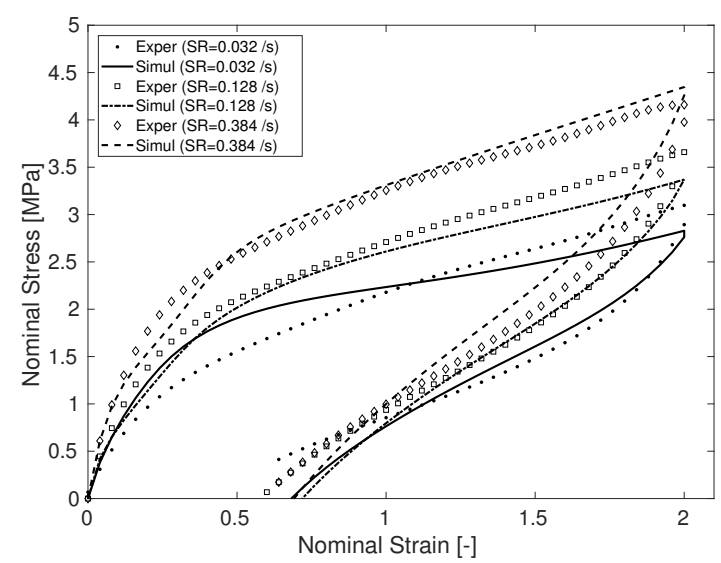

(b)

Figure 14. Model validation with strain rates of 0.032 /s, 0.128 /s, and 0.384 /s : (a) $100 \%$ strain, (b) $200 \%$ strain

results at lower strains and strain rates. In a further effort to provide validation for different strains at a particular strain rate, the results at 0.384 /s strain rate for strains ranging from $100 \%$ to $400 \%$ are shown in Fig. 15(b). The fittings clearly illustrate that the model can capture different types of data with a single set of parameters presented in Table 1.

\subsubsection{Validation by single- and multi-step relaxation data}

Finally, it is imperative to assess whether the identified same set of parameters can capture other timedependent viscous data. To this end, the remaining relaxation data are used for validation purposes. The validation with five data sets, i.e., 50\%, 100\%, 150\%, 200\%, and 250\% are presented in Fig. 16. Similar to the validation illustrated in the previous sections, the model nicely captures a wide spectra of data. Notably, the developed constitutive law closely predicts the total stresses, cf. Fig. 16. Additionally, it is validated with multi-step relaxation data and strains up to $350 \%$ (Fig. 17), which clearly illustrate that the model can predict various step changes of strains and corresponding stress relaxations. Note that, if we compare the validation with the loading-unloading cyclic data, the validation in the case of relaxation experiment deviates a bit from predicting the curved sections of the stress relaxation diagrams for the smaller strain cases. This could be improved using more strain and strain rate-dependent functions but it will unfortunately result in a much higher number of material parameters. However, at higher strains, the model nicely predicts the viscous overstresses observed in the experiments.

\section{Conclusion}

In this paper, for the first time, an additively manufactured elastomeric polyurethane (EPU) printed by the UV-curable DLS process is experimentally characterised. For this, all major characterization techniques suitable for a typical viscoelastic polymeric material are applied. These include stress-softening tests, quasistatic tests, cyclic tests, and relaxation tests. All time-dependent experiments demonstrate that EPU40 has a very strong strain rate-dependent behaviour that is idea for a viscoelastic polymer. The $3 \mathrm{D}$ printed polymer also shows a high stretchability with rupture occurring at strains above $600 \%$. Based on the experimental 


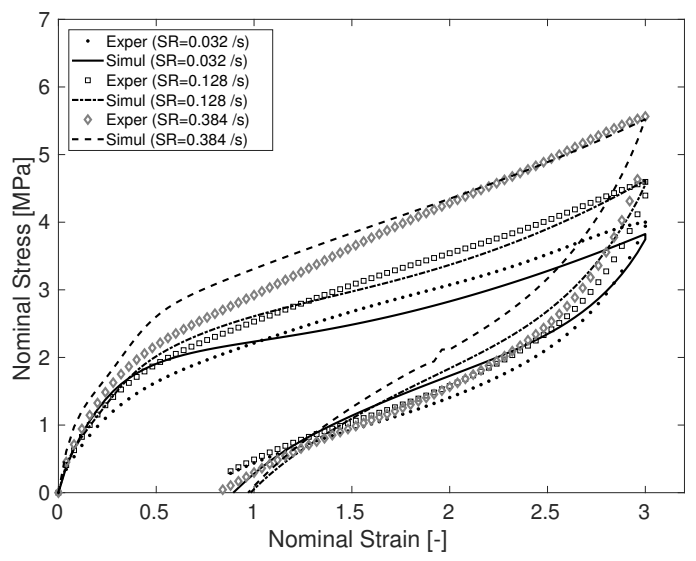

(a)

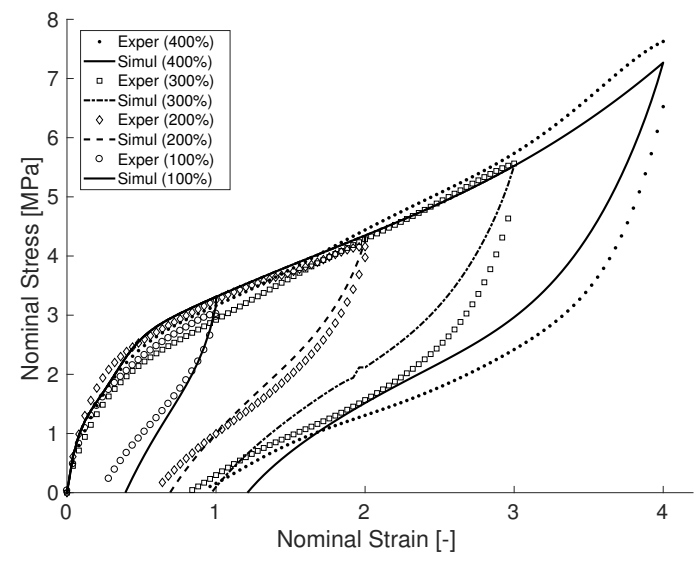

(b)

Figure 15. Model validation at different strains and strain rates: (a) 300\% strain at 0.032 /s, 0.128 /s, and 0.384 /s strain rates, (b) model validation with data ranging from $100 \%$ to $400 \%$ strains at a fixed strain rate of $0.384 / \mathrm{s}$.

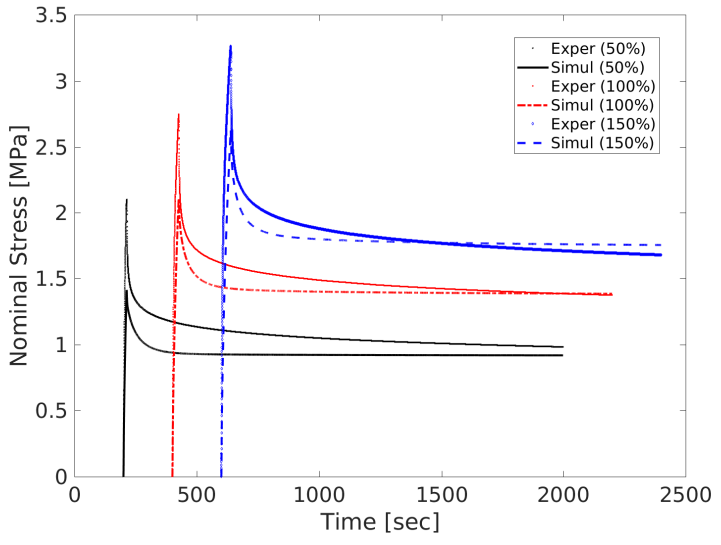

(a)

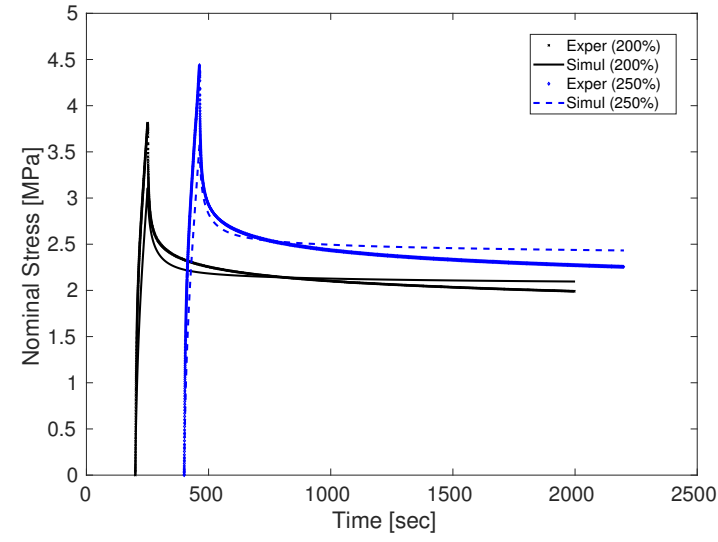

(b)

Figure 16. Model validation for relaxation data : (a) with the model for 50\%, $100 \%$ and $150 \%$ strains, (b) with the model for $200 \%$ and $250 \%$ strains 


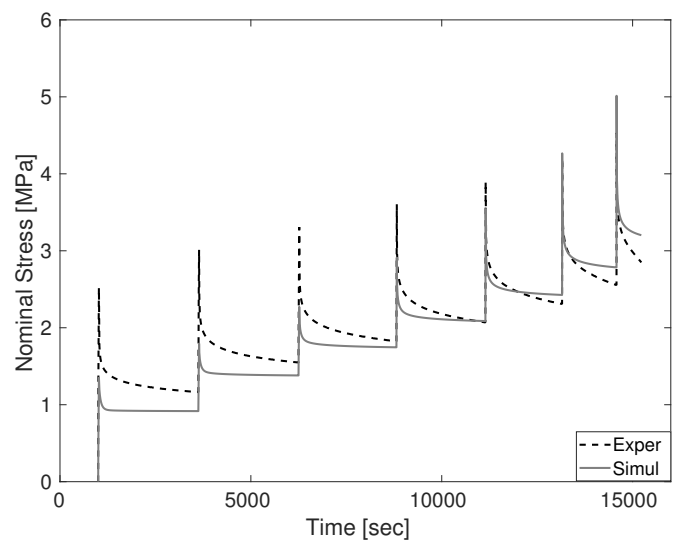

Figure 17. Model validation with multi-step relaxation data : Validated with experimental data up to $350 \%$ strain

study presented in the first half of the paper, we develop a constitutive model at finite strains that steps from the essential laws of thermodynamics. Using the uniaxial homogeneous tensile data, all material parameters of the proposed model are determined. The model is subsequently validated with several sets of new data. Model predictions illustrate good agreements with data obtained from a wide range of experiments. Owing to high tear strength, energy return, and a high elongation at break, the additively manufactured EPU is an attractive material choice for a wide range of applications including cushioning, impact absorption, vibration isolation, gaskets, and seals. Hence, a complete viscoelastic characterization and subsequent constitutive modelling of the material can be an essential tool in understanding constitutive response prior to making material selection for any particular application. Especially, the digital printing of soft polymers opens a new horizon of creating complex architectured cellular and metamaterials for which the 3D printed elastomeric polyurethanes such as EPU40 are potential candidates [49, 60].

\section{Acknowledgements}

Authors sincerely appreciate Colleges of Engineering at Swansea University and University of South Wales, UK for supporting this work with the Internal Grant Scheme (SURGE) and Impact Research Funding Scheme (IRFS), respectively.

\section{References:}

\section{References}

[1] R. Adams, S. P. Soe, R. Santiago, M. Robinson, B. Hanna, G. McShane, M. Alves, R. Burek, P. Theobald, A novel pathway for efficient characterisation of additively manufactured thermoplastic elastomers, Materials \& Design, $180: 107917,2019$

[2] A. F. M. S. Amin, A. Lion, S. Sekita and Y. Okui, Nonlinear dependence of viscosity in modeling the rate-dependent response of natural and high damping rubbers in compression and shear: Experimental identification and numerical verification, International Journal of Plasticity, 22:1610-1667, 2006 
[3] A. F. M. S. Amin, A. R. Bhuiyan, T. Hossain, Y. Okui, Nonlinear viscosity law in finite element analysis of high-damping rubber bearings and expansion joints, Journal of Engineering Mechanics, 141(6), 2015

[4] A.K. Bastola, M. Hossain, A review on magneto-mechanical characterizations of magnetorheological elastomers, Composites Part B: Engineering, In Press, 2020

[5] D. Blanco, P. Fernandez, A Noriega, Nonisotropic experimental characterization of the relaxation modulus for PolyJet manufactured parts, Journal of Materials Research, 29(17): 1876-1882, 2014

[6] Resin Data Sheets: CarbonResin EPU40, Carbon3D Company, https : / www. carbon3d.com/ materials/epu-elastomeric-polyurethane, 2018

[7] M. M. Carroll, A strain energy function for vulcanized rubbers, Journal of Elasticity, 103: 173-187, 2011

[8] J. C. Case, E. L. White, R. K. Kramer, Soft material characterization for robotic applications, Soft Robotics, 2(2):80-87, 2015

[9] G. Campoli, M. S. Borleffs, S. Amin Yavari, R. Wauthle, H. Weinansa, A. A. Zadpoor, Mechanical properties of open-cell metallic biomaterials manufactured using additive manufacturing, Materials \& Design, 49:957-965, 2013

[10] M. Cianchetti, C. Laschi, A. Menciassi, P. Dario, Biomedical applications of soft robotics, Nature Reviews, 3:143-153, 2018

[11] S. Cheng, B. Chen, Y. Zhou, M. Xu, Z. Suo, Soft sensor for full dentition dynamic bite force, Extreme Mechanics Letters, 34: 100592, 2020

[12] H. Cho. S. Mayer, E. Poeselt, M. Susoff, P. J.in 't Veld, G. C. Rutledge, M. C.Boyce, Deformation mechanisms of thermoplastic elastomers: Stress-strain behavior and constitutive modeling, Polymer, 128:87-99, 2017

[13] J. D. Davidson, N. C. Goulbourne, A nonaffine network model for elastomers undergoing finite deformations, International Journal of Mechanics and Physics of Solids, 61(3):1784-1797, 2013

[14] J. R. C. Dizon, A. H. Espera, Q. Chen, R. C. Advincula, Mechanical characterization of 3D-printed polymers, Additive Manufacturing, 20:44-67, 2018

[15] J. Diani, B. Fayolle, P. Gilormini, A review on the Mullins effect, European Polymer Journal, 45: 601612, 2009

[16] J.T. Fan, J. Weerheijm, L.J. Sluys, High-strain-rate tensile mechanical response of a polyurethane elastomeric material, Polymer, 65:72-80, 2015

[17] S. Ghorbanpour, M. E. Alam, N. C.Ferreri, A. Kumar, B. A. McWilliams, S. C.Vogel, J. Bicknell, I. J. Beyerlein, M. Knezevic, Experimental characterization and crystal plasticity modeling of anisotropy, tension-compression asymmetry, and texture evolution of additively manufactured Inconel 718? at 
[18] M. Hossain, D. K. Vu, P. Steinmann, Experimental study and numerical modelling of VHB 4910 polymer, Computational Materials Science, 59:65-74, 2012

[19] M. Hossain, P. Steinmann, More hyperelastic models for rubber-like materials: Consistent tangent operator and comparative study, Journal of the Mechanical Behaviour of Materials, 22(1-2):27-50, 2013

[20] M. Hossain, Z. Liao, An additively manufactured silicone polymer: Thermo-viscoelastic experimental study and computational modelling, Additive Manufacturing, 35: 101395, 2020

[21] G. A. Holzapfel, Nonlinear Solid Mechanics: A Continuum Approach for Engineering, Wiley \& Sons Ltd, UK, 2000

[22] K. Haldar, C. Pal, Rate dependent anisotropic constitutive modeling of brain tissue undergoing large deformation, Journal of the Mechanical Behavior of Biomedical Materials, 81:178-194, 2018

[23] M. Johlitz, H. Steeb, S. Diebels, A. Chatzouridou, J. Batal and W. Possart, Experimental and theoretical investigation of nonlinear viscoelastic polyurethane systems, Journal of Materials Science, 42:9894-9904, 2007

[24] C Kadapa, WG Dettmer, D Peric, Subdivision based mixed methods for isogeometric analysis of linear and nonlinear nearly incompressible materials, Computer Methods in Applied Mechanics and Engineering, 305:241-270, 2016

[25] N. Koprowski, M. Johlitz, S. Diebels, Characterizing the time dependence of filled EPDM, Rubber Chemistry and Technology, 84(2):147-165, 2011

[26] N. Koprowski, M. Johlitz, S. Diebels, Modelling of a cellular rubber with non-linear viscosity functions, Experimental Mechanics, 51:749-765, 2011

[27] Z. Liao, X, H. Yao, L. Zhang, M. Hossain, J. Wang, S. Zang, Temperature and strain rate dependent large tensile deformation and tensile failure behavior of transparent polyurethane at intermediate strain rates, International Journal of Impact Engineering, 129:152-167, 2019

[28] Z. Liao, M. Hossain, H. Yao, M. Mehnert, P. Steinmann, On thermo-viscoelastic experimental characterization and numerical modelling of VHB polymer, International Journal of Non-Linear Mechanics, 118:103263, 2020

[29] Z. Liao, M. Hossain, H. Yao, R. Navaratne, G. Chagnon Mehnert, A comprehensive thermo-viscoelastic experimental investigation of Ecoflex polymer, Polymer Testing, 86: 106478, 2020

[30] Z. Liao, M. Hossain, H. Yao, Ecoflex polymer of different Shore hardnesses: Experimental investigations and constitutive modelling, Mechanics of Materials, 144:103366, 2020

[31] L. Liu, Y. Li, A visco-hyperelastic softening model for predicting the strain rate effects of 3D-printed soft wavy interfacial layer, Mechanics of Materials, 137: 103128, 2019

[32] T. S. Lumpe, J. Mueller, K. Shea, Tensile properties of multi-material interfaces in $3 D$ printed parts, Materials \& Design, 162:1-9, 2019 
[33] S. C. Ligon, R. Liska, J. Stampfl, M, Gurr, R. Muelhaupt, Polymers for 3D printing and customized additive manufacturing, Chemical Reviews, 117:10212-10290, 2017

[34] J. Lizarazu, L. Goebel, S. Linne, S. Kleemann, T. Lahmer, Ch. Roessler, J. Hildebrand, Experimental characterization and numerical analysis of additively manufactured mild steel under monotonic

[35] A. T. Miller, D. L. Safranski, C. Wood, R. E. Guldberg, K. Gall, Deformation and fatigue of tough 3D printed elastomer scaffolds processed by fused deposition modeling and continuous liquid interface production, Journal of the Mechanical Behavior of Biomedical Materials, 75:1-13, 2017

[36] L. R. Meza, S. Das, J. R. Greer, Strong, lightweight, and recoverable three-dimensional ceramic nanolattices, Science, 345 (6202): 1322-1326, 2014

[37] M. Mehnert, M. Hossain and P. Steinmann, Numerical modeling of thermo-electro-viscoelasticity with field-dependent material parameters, International Journal of Non-Linear Mechanics, 126:13-25, 2018

[38] D. McCoul, S. Rosset, S. Schlatter, H. Shea, Inkjet $3 D$ printing of UV and thermal cure silicone elastomers for dielectric elastomer actuators, Smart Materials and Structures, 26 (12):1-17, 2017

[39] J. Mueller, K. Shea, C. Daraio, Mechanical properties of parts fabricated with injket $3 D$ printing through efficient experimental design, Materials \& Design, 86:902-912, 2015

[40] K. Morris, A. Rosenkranz, H. Seibert, L. Ringel, S. Diebels, F. E. Talke, Uniaxial and biaxial testing of 3D printed hyperelastic photopolymers, Journal of Applied Polymer Science, 137:48400, 2020

[41] L. Mullins, Softening of rubber by deformation, Rubber Chemistry and Technology, 42(1):339-362, 1969

[42] G. Machado, G. Chagnon and D. Favier, Theory and identification of a constitutive model of induced anisotropy by the Mullins effect, Journal of the Mechanics and Physics of Solids, 63:29-39, 2014

[43] D. Perić, W. Dettmer, A computational model for generalized inelastic materials at finite strains combining elastic, viscoelastic and plastic material behaviour, Engineering Computations, 20 (5-6): 768787, 2003

[44] H. J. Qi, M. C. Boyce, Stress-strain behavior of thermoplastic polyurethanes, Mechanics of Materials, $37: 817-839,2005$

[45] K. K. Reichl, D. J. Inman, Dynamic Mechanical and Thermal Analyses of Objet Connex 3D Printed Materials, Experimental Techniques, 42: 19-25, 2018

[46] M. Robinson, S. P. Soe, R. Johnston, R. Adams. B. Hanna, R. Burek, G. McShane, R. Celeghinie, M. Alves, $\mathrm{P}$. Theobald, Mechanical characterisation of additively manufactured elastomeric structures for variable strain rate applications, Additive Manufacturing, 27 :398-407, 2019

[47] T. Sheffer, F. Goldschmidt and S. Diebels, Implementation of the strongly pronounced non-linear viscoelasticity of an incompressible filled rubber, Technishe Mechanik, 35(2):118-132, 2015

[48] P. Saxena, M. Hossain and P. Steinmann P, A theory of finite deformation magneto-viscoelasticity, International Journal of Solids and Structures, 50 (24):3886-3897, 2013 
[49] A. Safar, L. A. Mihai, The nonlinear elasticity of hyperelastic models for stretch-dominated cellular structures, International Journal of Non-Linear Mechanics, 106:144-154, 2018

[50] K. Sedlan, Viscoelastisches Materialverhalten von Elastomerwerkstoffen, Experimentelle Untersuchung and Modellbildung (in German), PhD Dissertation, Universität Kassel, Germany, 2001

[51] P. Steinmann, M. Hossain and G. Possart, Hyperelastic models for rubber-like materials: Consistent tangent operators and suitability of Treloar's data, Archive of Applied Mechanics, 82(9):1183-1217, 2012

[52] Y. Su, B. Wu, W. Chen, M. Destrade, Finite bending and pattern evolution of the associated instability for a dielectric elastomer slab, International Journal of Solids and Structures, 158:191-209, 2019

[53] M. Simonelli, N. Aboulkhair, M. Rasa, M. East, C. Tuck, R. Wildman, O. Salomons, R. Hague, Towards digital metal additive manufacturing via high-temperature drop-on-demand jetting, Additive Manufacturing, 30: 100930, 2019

[54] V. Slesarenko, S. Rudykh, Towards mechanical characterization of soft digital materials for multimaterial 3D-printing, International Journal of Engineering Science, 123:62-72, 2018

[55] J. W. Stansbury, M. J.Idacavage 3D printing with polymers: Challenges among expanding options and opportunities, Dental Materials, 32(1): 54-64, 2016

[56] J. R. Tumbleston, D. Shirvanyants, N. Ermoshkin, R. Janusziewicz, A. R. Johnson, D. Kelly, K. Chen, R. Pinschmidt, J. P. Rolland, A. Ermoshkin, E. T. Samulski, J. M. DeSimone, Continuous liquid interface production of $3 D$ objects, Science, 347(6228), 2015

[57] A. Unkovskiy, S. Spintzyk, J. Brom, F. Huettig, C. Keutel, Direct 3D printing of silicone facial protheses: A preliminary experience in digital workflow, Journal of Prosthetic Dentistry, 120(2):303-308, 2018

[58] J.Yi, M. C. Boyce, G.F. Lee, E.Balizer, Large deformation rate-dependent stress-strain behavior of polyurea and polyurethanes, Polymer, 47(1):319-329, 2006

[59] S. Wang, S. A. Chester, Modeling thermal recovery of the Mullins effect, Mechanics of Materials, 126:88-98, 2018

[60] H. Wyatt, A. Safar, A. Clarke, S. L. Evans, L. A. Mihai, Nonlinear scaling effects in the stiffness of soft cellular structures, Royal Society Open Science, 6(1):181361, 2019

[61] M. Wissler, E. Mazza, Mechanical behaviour of an acrylic elastomer used in dielectric elastomer actuators, Sensors and Actuators A, 134:494-504, 2007

[62] T. J. Wallin, J. Pikul, R. F. Shepherd, 3D printing of soft robotic systems, Nature Reviews, 3:84-100, 2018

[63] T. T. Wohlers, T. Caffrey, R. I. Campbell, Wohlers report 2016:3D printing and additive manufacturing state of the industry : annual worldwide progress report, Wohlers Report, 2016

[64] J. Zhou, L. Jiang, R. E. Khayat, A micro-macro constitutive model for finite-deformation viscoelasticity of elastomers with nonlinear viscosity, Journal of the Mechanics and Physics of Solids, 110:137-154, 2018 